\title{
1 Measuring heterogeneity in soil networks: a network analysis and
}

2 simulation-based approach

3

4 Natalie Davis ${ }^{a, b}$, *

5 J. Gareth Polhill b

6 M. J. Aitkenhead ${ }^{b}$

7 a Lancaster Environment Centre, Lancaster, UK.

$8{ }^{\mathrm{b}}$ The James Hutton Institute, Aberdeen, UK.

$9 *$ Corresponding author: ndavis.research@gmail.com

\section{Abstract}

Quantifying soil structural and ecological heterogeneity is crucial for understanding their interactions and their relationships to the resilience and health of the wider ecosystem. However, a clear understanding of how structural heterogeneity affects soil biodiversity is still emerging. Previous work has primarily used expensive, often laboratory-based methods to quantify soil pore network structure, and typically separated study of structural and biological dimensions. Here, we test whether standard network metrics can be used to quantify structural heterogeneity in soil pore networks, and how this network structure, along with characteristics of the consumer and resource populations, affects the heterogeneity of a population of consumers. Specifically, we extract simplified soil pore networks from digital photographs of soil profiles and apply established metrics from network science and transport geography to quantify and compare the networks. The networks are also used as the medium for an agent-based model of generalised consumers, to analyse the effects of consumer and resource parameterisations and network structure. Combining network analysis and simulation modelling in this way can provide insights on the structure, function, and diversity possible in the soil, as well as avenues for exploring the impact of future structural or environmental changes.

Keywords: soil networks; soil structure; digital soil morphometrics; agent-based model; network analysis; ecological heterogeneity. 


\section{Introduction}

The distribution of energetic resources in an ecosystem plays a key role in determining the complexity, quantity, and behaviour of organisms that it can support (e.g. Giller, 1996; Tews et al., 2004; Roshier, Doerr and Doerr, 2008; Stevens \& Tello, 2011). To understand these systems more fully, and inform actions to protect those relying on them, we must understand how resource distribution networks develop and function. For example, resource location and movement can create heterogeneity that allows species to specialise and differentiate (e.g. Bardgett, Yeates and Anderson, 2009; Tews et al., 2004; Stevens \& Tello, 2011), as well as cause inequality among individuals of the same species, topics that are relevant for both biologists and ecologists.

The soil provides a unique and diverse ecosystem in which to study resource distribution, and its effect on organisms. Soil structure can be defined as the collection of soil particles and pore space among them (Oades, 1993). This pore space provides access to nutrients stored on the surface of soil particles, allows for preferential flow of water through the soil matrix, and serves as the resource distribution network through which micro-, meso-, and macrofauna (soil biota) forage. As this structure determines how air, water, and soil biota move through the soil, it allows or impedes the foraging of organisms, regulates the air and water balance in the soil matrix, and affects chemical signals used in foraging, such as those of bacterial decomposition (Young and Ritz, 2009). Furthermore, crevices and niches along soil pores provide habitats for smaller microbes to avoid predation, and the overall spatial and temporal heterogeneity of the soil environment allows for resource partitioning and habitat specialisation that limits the effect of competitive exclusion (Bardgett, Yeates and Anderson, 2009). This is similar to the hypothesised effect of heterogeneity in aboveground habitats (e.g. Tews et al., 2004; Stevens and Tello, 2011).

Soil biota in turn can increase the porosity of soil, through burrowing and consuming organic matter, and releasing gases during decomposition, which create or expand soil pores (Kravchenko and Guber, 2017). Additionally, there is evidence of feedbacks between the soil biota and aboveground plant communities (e.g. Baer et al., 2005; Wijesinghe, John and Hutchings, 2005; García-Palacios et al., 2012), which alter soil structure as their roots burrow in pore networks, and roots and hyphae bind and stabilise soil particles (Vezzani et al., 2018). Through regulating movement and diffusion of water and energy resources, gases, and fauna in the soil matrix; providing habitat; and mediating biological feedbacks; soil structure is the foundation of all earth systems.

Past efforts to quantify and model soil structure have primarily focussed on measuring the stability of soil, by utilising soil aggregate size distribution as a measure of structure. While this does represent the spatial distribution in the soil, it is not a complete representation of physical properties (see e.g. Young, Crawford and Rappoldt, 2001). Methods for visualising the pore network within a soil sample include CT scans and X-ray tomography, NMR, and SPECT scanning, mostly for the purposes of measuring solute flow and transport processes (see review in Young, Crawford and Rappoldt, 2001). Gas diffusion and solute flow have also been examined with modelling approaches, including neural networks, Boolean models, and cellular automata. Additionally, fractal modelling has also been used successfully to quantify the degree of connectivity, tortuosity, and heterogeneity of the soil pore network (Crawford, Ritz and Young, 1993), three characteristics that have also been associated with a higher level of heterogeneity of resource distribution in generalised networks (Davis et al., 2020).

Overall, past work has highlighted the important connections between soil function and structure, especially of the pore network. Much of this work has been done from a geometric or hydrological perspective, however, rather than an energetic one, leading to criticisms of unrealistic separation of 
soil physics and biology, and emphasis on the importance of integrating these spatially explicit approaches in future soil ecology research (Bardgett, Yeates and Anderson, 2009). Additionally, much of the imaging equipment required for the techniques above is large and expensive, requiring soil samples to be brought back to the laboratory. Even if disruption to the soil structure during extraction and transport is minimised, these methods are more suitable for intensive analyses of individual samples and smaller areas.

In contrast, some previous work has focussed on quantifying the structure of soil networks through image morphology techniques applied to a photograph of a sample, in order to extract the relevant network (e.g. Velde, Moreau and Terribile, 1996; Gargiulo, Mele and Terribile, 2013; Hartemink and Minasny, 2014). This method will not reveal the network at the same level of detail as CT scans or Xray tomography, and may require use of resins and dyes to highlight the underlying structure (Hartemink and Minasny, 2014). Good arguments have also been raised regarding the importance of analysing soil structure from a three-dimensional perspective, as it reveals considerably more about the habitat of the soil (Young and Ritz, 2009). However, if rotational invariance is assumed, connectivity and structure of a two-dimensional sample can be assumed representative of any random two-dimensional plane taken through the system. This inference does not consider lateral flow, which would undoubtedly play an important influence in sloping areas by transporting nutrients laterally through the soil. In areas where the surface is flat and lateral flow effects are negligible, standard network metrics could usefully approximate soil structure and provide insights into its effect on biotic and abiotic processes within an environment.

Moreover, the two-dimensional techniques are considerably more portable and feasible than the three-dimensional techniques, and processing time can be significantly faster. Image analysis methods, particularly those that can be performed entirely in the field, could potentially be incorporated into software for use by farmers and researchers who may otherwise not have access to the equipment necessary for the more costly and lab-intensive methods of quantifying structure (e.g. Aitkenhead et al., 2016). These methods could also act as preliminary investigations to highlight potential areas of future exploration using more intensive analyses.

In this paper, we test whether standard network metrics can be used to quantify structural heterogeneity in soil pore networks, and how this network structure, along with characteristics of the consumer and resource populations, affects the heterogeneity of a population of consumers. Specifically, we develop a method for extracting approximate soil networks from digital photographs using image morphology techniques, then apply metrics from network science and transport geography to quantify and compare the networks. The networks are also used as the medium for an agent-based model (ABM, which in ecology is more typically known as an individual-based model, e.g. Grimm et al., 2006), where the agents represent generalised consumers who explore the network and consume food resources. The variation in population size and resource consumption is compared across simulations, to evaluate how both the network structure and simulation parameters affect outcomes of the biotic community. This methodology is applied to a case study using soil images from two test sites in Aberdeenshire, United Kingdom. 


\subsection{Soil image collection}

118 Images were taken at two field sites in Aberdeenshire, United Kingdom. The first site had a brown forest soil, or Cambisol (Fig A1a); photographs were taken from seven locations in both forested and converted agricultural areas. The second site had a sandy beach soil, or Arenosol (Fig A1b); photographs were taken at five locations across a dune area, with sparse grass and shrub cover. Neither Cambisols nor Arenosols are highly developed, but Cambisols have some diagnostic features, while Arenosols are lacking diagnostic features and are defined only on the basis of being coarse (sandy) textured (FAO, 2015). The known difference between the two soils therefore provides a show limited profile variation with depth on the scale of the observed soil profile sections under study (FAO, 2015), such that a uniform network extraction method and analysis can be applied across the image. The specific sampling sites were also chosen as they provided easy access to multiple sampling locations for both soil types. As this work is an exploratory proof-of-concept, an exhaustive sampling regime across different soil, land use, and geographic regions was not undertaken.

The methodology for taking pictures was replicated from Aitkenhead et al. (2016). In summary, the photographs were taken of the soil profile of shallow $(30 \mathrm{~cm})$ pits in flat areas, using an angle that provided maximum natural light and minimum shadow (Fig A1a, b). No artificial lighting was required during photography. Additionally, each photograph included a $10 \mathrm{~cm} \times 6 \mathrm{~cm}$ colour correction card within the frame. Colour correction has been used in past work (e.g. Aitkenhead et al., 2016) to correct colour variation in ambient lighting. However, in this work we were only interested in overall intensity, rather than light balance, so the cards were inserted into the image to provide a spatial scale reference for future work.

In Fig A1a and A1b, the white area is an excised section of the image that is larger than the correction card. The imaging was taken with the card viewed straight on, without distortion, so the image distortion and impact on length of edges is not an issue. Extracting an area larger than the correction card also attempted to eliminate shading effects around the card. This may not have been done sufficiently to eliminate all the shading, possibly introducing some additional dark pixels and error into the network metric calculations. However, taking multiple pictures within the same profile can provide some robustness against this. Future work should attempt to remove this effect from near the correction card.

In total, seven Cambisol profiles and five Arenosol pits were used for each soil type, with several images taken of the profile of each pit. In taking multiple images from each soil pit, we moved the camera slightly to present different viewing angles and thus generate different images. This was done to compare the robustness of extracted networks from each pit (see Section 2.2), and replication within pit was considered in all statistical analyses.

\subsection{Network extraction}

To extract the approximate soil network structure from the photographs, the photographs were converted to text files containing the red, green, and blue (RGB) triplet values for each pixel. All nonsoil pixels were then identified as those whose triplet values exceeded the ranges expected for soil particles, based on the average of the rest of the image. Using the average to determine this threshold customised it slightly for each image, so that outliers such as roots and rocks specific to that sample were captured, but samples having an overall more reddish tone were not stripped 
completely. The identified non-soil particles were removed, and variations in brightness across the remaining pixels were standardised using the mean pixel intensity.

As soil structure and porosity are only loosely related, soils of the same porosity can have different structural properties. A common assumption made is that soils, unless compressed/compacted, have up to $50 \%$ pore space. As the pixel resolution of the images here is between $0.3-0.5 \mathrm{~mm}$, and therefore much higher than the smallest pore space possible (sub-micron scale), it follows that the pore space actually visible is less than this $50 \%$. An evaluation of the distribution of pixel values showed that for soil profile images used in this study, the greatest change in the distribution occurred around a pixel intensity where $30-40 \%$ of the pixels were below this value (Fig A2). We have therefore assumed that $30 \%$ of the soil is 'void' (i.e. dark pixels). Therefore, the darkest $30 \%$ of the soil pixels were retained as pores, and the image was inverted to convert these darker pixels to white, and vice versa (Fig A1e, f). The images from the same profile were visually compared after thresholding and showed a high degree of agreement in the pores identified (e.g. Fig A3). Network outlines were then drawn through a process known in image morphology as 'skeletonization,' where lines of white pixels were iteratively stripped down until they were all one pixel in width (Fig. A1g, h). We then mapped the networks to a list of links, which were series of pixels that were more than one pixel long, and nodes, defined as junction points between two or more links. Redundant links between nodes were removed.

For simplicity, all links in the final networks were represented with straight lines along the shortest distance between two nodes. This lost some of the details of the topology, such as pore size and shape. However, this work intended to create an abstraction of the network taken from the soil, rather than replicate and analyse the exact soil structure itself. This emphasised overall soil structural characteristics and heterogeneity, rather than modelling how specific transport processes and biological activities would occur. Replicating the exact soil network would also have markedly increased the computational burden, as link lengths would have had to be calculated through pixelcounting rather than the Euclidean geometry measuring shortest distances. As many of the links as represented were quite short (see Results), the difference between the true link length and the shortest distance between nodes was assumed to be negligible. Currently, we assume that the method requires further validation and improvement to provide a measure of soil structure that can be used in soil science or pedological characterisation of the soil. We also assume however, that the method, while not perfect in its current form, provides sufficient quality of network data to allow simplified networks to be extracted and analysed, and used as the basis for simulations.

The process of rendering the network also identified which sections of the network were fully connected, and which nodes were part of disconnected subnetworks (Fig. A1i, j). An outline of the image morphology process, and images of each step, are available in Appendix 1.

\subsection{Network analysis}

Two types of analysis were used to quantify the heterogeneity present in the soil network images. The first involved applying metrics adapted from network science and transport geography to measure structural characteristics of the abstracted networks, which allows for easy comparison among soil types. These were calculated using R v4.0.2 (R Core Team, 2020), including the packages igraph, qgraph, and sp (Pebesma and Bivand, 2005; Csardi and Nepusz, 2006; Epskamp et al., 2012; Bivand, Pebesma and Gomez-Rubio, 2013). All additional data analysis and visualisations were also done in R, using the packages ARTool v0.10.7 (Kay and Wobbrock, 2020; Wobbrock et al., 2011), emmeans v1.5.0 (Lenth, 2020), ImerTest v3.1.2 (Kuznetsova, Brockhoff, and Christensen, 2017), dunn.test v1.3.5 (Dinno, 2017), rcompanion v2.3.25 (Mangiafico, 2020), dplyr v1.0.0 and ggplot2 
v3.3.2 packages (Wickham, 2016; Wickham et al., 2019). The scripts for calculating network metrics are available at (Davis, 2020).

A brief description of each of the metrics chosen is given in Table 1. These were chosen to measure the size, connectivity, and structural heterogeneity of the networks from a range of node-centric, link-centric, and global perspectives, to obtain a broad picture how the networks may differ. The metrics chosen also minimised assumptions about inaccessibility of the soil matrix between pores: for example, the convex hull area was chosen over the concave hull area as the former is a more generous estimate of the spatial area.

Table 1. The name and description of the metrics used to analyse the soil networks.

\begin{tabular}{|c|c|c|c|}
\hline Metric name & Description & $\begin{array}{l}\text { Type of } \\
\text { measure }\end{array}$ & Reference \\
\hline $\begin{array}{l}\text { Mean and standard } \\
\text { deviation (SD) of link } \\
\text { length }\end{array}$ & $\begin{array}{l}\text { Quantifies the typical length and variability } \\
\text { of lengths included within the network. }\end{array}$ & Size & $\mathrm{N} / \mathrm{A}$ \\
\hline Beta index & The ratio of links to nodes. & Connectivity & $\begin{array}{l}\text { Rodrigue, } \\
2017\end{array}$ \\
\hline Gamma index & $\begin{array}{l}\text { Number of observed vs. possible links: } \\
\text { nLinks / (nNodes * (nNodes }-1) \text { ) }\end{array}$ & Connectivity & $\begin{array}{l}\text { Rodrigue, } \\
2017\end{array}$ \\
\hline Diameter & $\begin{array}{l}\text { The length of the longest geodesic (shortest } \\
\text { path between two nodes) in the network - } \\
\text { the shortest path between the two most } \\
\text { distant nodes. }\end{array}$ & Size & $\begin{array}{l}\text { Rodrigue, } \\
2017\end{array}$ \\
\hline Node count & The number of nodes in the network. & Size & $\begin{array}{l}\text { Barabási, } \\
2016\end{array}$ \\
\hline Edge count & The number of edges (links) in the network. & Size & $\begin{array}{l}\text { Barabási, } \\
2016\end{array}$ \\
\hline Mean node degree & Mean number of links per node. & Connectivity & $\begin{array}{l}\text { Barabási, } \\
2016\end{array}$ \\
\hline Cost & $\begin{array}{l}\text { The total length of the network measured in } \\
\text { real transport distances. }\end{array}$ & Size & $\begin{array}{l}\text { Rodrigue, } \\
2017\end{array}$ \\
\hline $\begin{array}{l}\text { Global reach } \\
\text { centrality (GRC) }\end{array}$ & $\begin{array}{l}\text { The difference between the maximum and } \\
\text { average local reach centrality (LRC), where } \\
\text { the LRC is the nodes that a given node can } \\
\text { connect to, weighted by distance (here, } \\
\text { spatial distance). }\end{array}$ & $\begin{array}{l}\text { Structure, } \\
\text { connectivity }\end{array}$ & $\begin{array}{l}\text { Adapted from } \\
\text { Mones, } \\
\text { Vicsek and } \\
\text { Vicsek (2012) }\end{array}$ \\
\hline $\begin{array}{l}\text { Mean convex hull } \\
\text { area }\end{array}$ & $\begin{array}{l}\text { The area of a polygon that minimally } \\
\text { encompasses every node in the network. }\end{array}$ & $\begin{array}{l}\text { Size, } \\
\text { connectivity }\end{array}$ & $\begin{array}{l}\text { Rockafellar, } \\
1970\end{array}$ \\
\hline Network density & $\begin{array}{l}\text { The ratio of the number of nodes to the } \\
\text { convex hull area. }\end{array}$ & Structure & N/A \\
\hline
\end{tabular}


As introduced, the imaging method and metrics used here are two-dimensional (2D), and we have been unable to find literature describing characterisations of three-dimensional (3D) soil structure metrics based on two-dimensional imaging. Aitkenhead et al. (1999) derived 3D models of soil pore systems based on 2D metrics but did not compare the two sets of structural metrics. Future work would be necessary to determine the extent to which 3D variation in soil structural metrics correlates to the variation seen in 2D. Here, we are assuming that it does correlate, and that this allows $2 \mathrm{D}$ imaging to provide structural metrics representative of different soil types.

We calculated each metric for each of the networks, which contained all nodes and links in the image, hereon called 'main networks.' We also calculated each metric for each of the disconnected subnetworks within the main networks, hereon called 'subnetworks.' As the distributions of metrics in the main soil networks had similar variance across soil types and relatively normal distributions, these were compared with nested ANOVA, using profile ID as a random effect to account for replication. The distribution of metrics across the subnetworks did not meet the assumptions for classical ANOVA, so non-parametric Aligned-Ranks Transformation (ART) ANOVAs were used instead, also with profile ID as a random effect.

\subsection{Agent-based model overview}

The second analytical method used a simulated population of consumers to explore each network, using the resulting heterogeneity in consumer resource stocks to further elucidate the heterogeneity of the network. This provided a more functional perspective, alongside the structural quantification of the network metrics. The purpose was to investigate the structure's generalised impact, rather than test the precision of this model in predicting outcomes for real species. Therefore, rather than using parameterisations that reflected specific species or groups, five generic model species with different sets of values for each trait were used, similarly to e.g. Polhill and Gimona (2014).

The same approach was taken for resources, with three sets of resource bases of different combinations of maximum capacity and maximum growth rates. Resources were assumed to be located at nodes within the network, as identified during the extraction process (see Section 2.2). Food resources in real soil networks are located throughout the soil matrix, but are often concentrated in 'hotspots' such as those created by plant roots and decomposition processes (Ettema and Wardle, 2002), which would be represented in the networks here as nodes. As exploring the effect of size of the generic species was not in scope for the work here, only the most accessible areas of the network were treated as potential resources.

A brief description of the model purpose, variables, and processes is presented below, following the Overview, Design concepts, and Details (ODD) protocol (Grimm et al., 2006, 2010). The full ODD document, including description of design concepts, initialisation, input data, and sub-models, is available in Appendix 2. The model source code, written in NetLogo 6.1, is available in the Modelling Commons repository as "Soil network simulation" (Davis and Polhill, 2020).

\subsubsection{Overview section of Overview, Design Concepts, and Details (ODD)}

\section{Model purpose}

a. The model is designed to be an analytical tool to explore the heterogeneity in resource supply potential of a network by populating it with idealised energyconsuming agents, and to quantify the effects of consumer, resource, and network characteristics on resulting consumer population outcomes.

II. Entities, state variables, and scales

a. Consumer entities 


\begin{tabular}{ll}
\hline Property & Description \\
\hline Location & The resource on which the consumer is located \\
Target location & The resource to which the consumer will move next \\
Active? & Whether a consumer is active (or dead) \\
\hline
\end{tabular}

ii. Parameters

Property Description

Basal metabolism How much resource an agent needs per day to stay alive

Active metabolism How much resource an agent uses with each step

Resource stock How much resource an agent has consumed but not metabolised

Consumption rate Maximum number of resource units that an agent takes from a resource it visits, per timestep

Spawn energy How much energy an agent requires to spawn (depletes this quantity from stocks and passed to offspring as starting quota)

260

\section{Property}

b. Resource entities

Description

ii. Parameters

c. Link entities

d. Scales

i. State variables

\begin{tabular}{ll}
\hline Property & Description \\
\hline Resource capacity & How much energy is stored in a resource when it is full \\
Regrow rate & The amount the resource regrows each timestep \\
\hline
\end{tabular}

\begin{tabular}{ll}
\hline Property & Description \\
\hline Length & The length of the link - determines energy and time required to traverse it \\
\hline
\end{tabular}

\begin{tabular}{ll}
\hline Property & Description \\
\hline Timestep & $\begin{array}{l}\text { A single unit of time in the model, defined as that which is required for } \\
\text { consumers to move } 1 \text { pixel (approximately } 0.3-0.5 \mathrm{~mm} \text { ), and for which they } \\
\text { require basal-metabolism units of energy. }\end{array}$ \\
$\begin{array}{l}400 \times 500, \text { determined by the size of the soil networks used as the } \\
\text { environment. }\end{array}$ \\
\hline
\end{tabular}


a. Consumers start on random nodes around a pre-specified network, where nodes are resource patches.

b. Consumers move around the network randomly following links. If they find a resource patch, they consume as much as they can from it, and the patch depletes.

i. Consumers require basal-metabolism units of resource per timestep. If they do not consume this resource, they die.

ii. Consumers can stay put on a resource and consume it (consumptionrate units consumed per timestep), but it depletes, and if there is no more resource there then they move on.

iii. Consumers metabolise active-metabolism units of resource per patch of link that they cross.

iv. If there is more than one agent on a resource patch, they each take consumption-rate units per timestep, or split the remainder if there is not enough resource remaining for them to each get consumption-rate units.

c. If consumers have twice as much energy as the set spawn-energy, they can spawn new consumers (who take the same amount of resource-stock from their parent that the parent started with, so now parent and offspring both have the same resource-stock).

d. Resources regrow at a constant rate (regrow-rate) per timestep, up to their maximum capacity (resource-capacity).

\subsubsection{Sensitivity analysis}

To determine the sensitivity of the ABM to input parameters, and the robustness of any emergent patterns of heterogeneity, we performed an extensive sensitivity analysis following recommendations in the agent-based modelling literature. This is detailed in Appendix 3. Table 2 shows the final parameter values used for the consumer populations, resource populations, and general model. In the actual simulation runs, each combination of the five consumer parameter sets, and three resource parameter sets, was tested against each network architecture, resulting in 8700 total runs including replicates.

Table 2. Final values for (a) consumer, (b) resource, and (c) general simulation parameters.

a. Consumer parameters

\begin{tabular}{|c|c|c|c|c|c|}
\hline \multirow[b]{2}{*}{ Parameter } & \multicolumn{5}{|c|}{ Consumer type } \\
\hline & $\begin{array}{c}\text { High } \\
\text { metabolism, } \\
\text { high } \\
\text { consumption, } \\
\text { high spawn } \\
\text { energy } \\
\text { (HHH) }\end{array}$ & $\begin{array}{c}\text { Low } \\
\text { metabolism, } \\
\text { low } \\
\text { consumption, } \\
\text { low spawn } \\
\text { energy } \\
\text { (LLL) }\end{array}$ & $\begin{array}{l}\text { Low } \\
\text { metabolism, } \\
\text { moderate } \\
\text { consumption, } \\
\text { low spawn } \\
\text { energy (LML) }\end{array}$ & $\begin{array}{c}\text { Low } \\
\text { metabolism, } \\
\text { moderate } \\
\text { consumption, } \\
\text { moderate } \\
\text { spawn energy } \\
\text { (LMM) }\end{array}$ & $\begin{array}{l}\text { Moderate } \\
\text { metabolism, } \\
\text { moderate } \\
\text { consumption, } \\
\text { moderate } \\
\text { spawn energy } \\
\text { (MMM) }\end{array}$ \\
\hline Basal metabolism & 3 & 1 & 1 & 1 & 2 \\
\hline Active metabolism & 3 & 1 & 1 & 1 & 2 \\
\hline Consumption rate & 10 & 5 & 7 & 7 & 7 \\
\hline Spawn energy & 100 & 50 & 50 & 75 & 75 \\
\hline Initial resource stock & 30 & 30 & 30 & 30 & 30 \\
\hline
\end{tabular}




\begin{tabular}{lcccc}
\cline { 2 - 5 } & \multicolumn{4}{c}{ Resource type } \\
\hline Parameter & $\begin{array}{c}\text { High capacity, } \\
\text { low growth } \\
\text { (HL) }\end{array}$ & $\begin{array}{c}\text { Moderate capacity, } \\
\text { moderate growth } \\
\text { (MM) }\end{array}$ & $\begin{array}{c}\text { Low capacity, high } \\
\text { growth } \\
\text { (LH) }\end{array}$ \\
\hline Maximum resource capacity & & 50 & 35 & 20 \\
Maximum regrow rate & & 10 & 15 & 20 \\
\hline
\end{tabular}

305

c. General parameters

\begin{tabular}{ll}
\hline Parameter & Value \\
\hline Initial population size & 500 consumers \\
Length of simulation & 2000 timesteps \\
\hline
\end{tabular}

307

\subsubsection{Analytical method}

309 At each time step, the ABM calculated five metrics (Table 3), including measures of centre and

310 spread of consumer resource stocks, the final population size, and two additional inequality metrics:

311 the Gini coefficient and a modified form of the Shannon entropy. The latter estimates the

312 differential entropy of a continuous variable, by discretising the distribution into bins (Appendix 4).

313 These metrics were chosen to include measures of absolute and relative inequality, and a measure

314 of evenness common to ecology. As the distributions of each metric across the soil types did not

315 meet assumptions of most parametric tests, mixed-effects ART ANOVAs with profile ID as a random

316 effect were again used to quantify how the outcome metrics differed, for each combination of

317 resource and consumer population parameters and soil type. As the final population size and the

318 entropy of consumer resource stocks both showed variance not fully explainable by consumer or

319 resource population parameters, these were also tested with Kruskal-Wallis tests comparing them

320 across profile IDs and soil types. The significantly different pairs of profiles were identified with Dunn

321 post-hoc analysis. All data processing, analysis, and visualisation was done in $\mathrm{R}$, using the packages listed previously, as well as the entropy v1.2.1 (Hausser and Strimmer, 2014) and ineq v0.2.13

323 packages (Zeileis, 2014). 

(ABM).

\begin{tabular}{|c|c|}
\hline Variable name & Description \\
\hline $\begin{array}{l}\text { Mean consumer } \\
\text { resource stock }\end{array}$ & $\begin{array}{l}\text { The mean of the resource stocks held by all active consumers. Units are } \\
\text { the same as those of the quantity measured. }\end{array}$ \\
\hline \multirow[t]{2}{*}{$\begin{array}{l}\text { Standard deviation } \\
\text { consumer resource } \\
\text { stock }\end{array}$} & $\begin{array}{l}\text { The square root of the sum of squared absolute differences between } \\
\text { each observation and the mean, normalised by the number of } \\
\text { observations (minus one, to allow for sample estimation). Units are the } \\
\text { same as those of the quantity measured. }\end{array}$ \\
\hline & $s=\sqrt{\frac{\sum\left|x_{i}-\bar{x}\right|^{2}}{n-1}}$ \\
\hline $\begin{array}{l}\text { Gini coefficient } \\
\text { consumer resource } \\
\text { stock }\end{array}$ & $\begin{array}{l}\text { Measures the deviation of a population from perfect equality. } \\
\text { Mathematically, it can be calculated as half the relative mean absolute } \\
\text { difference, or half the average absolute difference between all pairs of } \\
\text { the population, divided by the average of the population to normalise. } \\
\text { Unitless. }\end{array}$ \\
\hline $\begin{array}{l}\text { Entropy consumer } \\
\text { resource stock } \\
\text { (Shannon index) }\end{array}$ & $\begin{array}{l}\text { Measures the amount of information that would be needed to } \\
\text { represent the state of the system. Specifically, it is the negative sum of } \\
\text { the probability of a consumer's resource stock occurring within a given } \\
\text { range, and the log of that probability, normalised by the maximum } \\
\text { value (log } n) \text {. This is the discretised formula for entropy. The units } \\
\text { depend on the base of the log: here we use base } 2 \text { (units: bits). } \\
\qquad H(X)=\frac{-\sum f\left(x_{i}\right) \log _{2} \frac{f\left(x_{i}\right)}{w\left(x_{i}\right)}}{H_{\max }}\end{array}$ \\
\hline Final population size & Count of currently active ('alive') consumers. \\
\hline
\end{tabular}




\section{Results}

\subsection{Network metrics}

330 The network metrics showed several significant differences between the Cambisols and Arenosols, with the Cambisols having higher values for most metrics measuring size and structure. These are summarised in Table 4 and Fig 1.

Table 4. (a) Estimated marginal means, standard errors, and outcomes for mixed-effect nested ANOVAs comparing network metrics between Cambisol and Arenosol main soil networks, and (b) medians and $95 \%$ confidence intervals and results of mixed-effect nested Aligned-Ranks Transformation (ART) ANOVAs comparing Cambisol and Arenosol subnetworks. Shown in (a) are the Type II Wald Chi-square statistic and $p$-values for models comparing each network metric across soil types. Profile ID was included as a mixed effect; its log-likelihood ratio test (LRT) statistic and $p$ value are also shown. Both the Chi-square and LRT used one degree of freedom to compare soil types. Estimated marginal means and standard errors were calculated from ANOVAs. In (b) ART ANOVAs were used as the data were non-normal; profile ID was also included as a mixed effect. Shown are Type III Wald F tests with Kenward-Roger degrees of freedom. The asterisks designate level of significance: $p<0.1: \cdot, p<0.05:^{*}, p<0.01:^{* *}, p<0.001:{ }^{* * *}$. Descriptions of the metrics are in Table 1. 


\begin{tabular}{|c|c|c|c|c|c|c|c|}
\hline & \multicolumn{2}{|c|}{$\begin{array}{c}\text { Arenosols } \\
(n=25)\end{array}$} & \multicolumn{2}{|c|}{$\begin{array}{c}\text { Cambisols } \\
(n=25)\end{array}$} & \multicolumn{2}{|c|}{ Soil type } & Profile ID (random effect) \\
\hline & $\begin{array}{l}\text { Est. marginal } \\
\text { mean }\end{array}$ & SE & $\begin{array}{c}\text { Est. marginal } \\
\text { mean }\end{array}$ & SE & $\chi^{2}$ & $p$ & LRT \\
\hline No. of nodes & 2670.000 & 9.570 & 3326.000 & 10.070 & 7.809 & $0.005 * *$ & $21.384<0.001 * * *$ \\
\hline No. of links & 4225.000 & 344.000 & 5581.000 & 293.000 & 9.058 & $0.003 * *$ & $19.280<0.001 * * *$ \\
\hline Mean node degree & 3.140 & 0.055 & 3.350 & 0.047 & 9.098 & $0.003 * *$ & $9.0050 .003 * *$ \\
\hline Mean link length & 3.570 & 0.023 & 3.700 & 0.022 & 17.667 & $0.000 * * *$ & 0.4140 .520 \\
\hline SD link length & 2.110 & 0.025 & 2.210 & 0.025 & 9.815 & $0.002 * *$ & 0.0001 .000 \\
\hline Gamma index & 0.001 & 0.000 & 0.001 & 0.000 & 4.838 & $0.028 *$ & $20.231<0.001 * * *$ \\
\hline Beta index & 1.570 & 0.028 & 1.680 & 0.024 & 9.098 & $0.003 * *$ & $9.0050 .003 * *$ \\
\hline Diameter & 154.000 & 11.100 & 201.000 & 10.700 & 10.017 & $0.002 * *$ & 0.1410 .708 \\
\hline Cost & 15180.000 & 1257.000 & 20600.000 & 1072.000 & 10.840 & $0.001 * * *$ & $16.153<0.001 * * *$ \\
\hline $\begin{array}{l}\text { Global reach } \\
\text { centrality }\end{array}$ & 0.002 & 0.000 & 0.002 & 0.000 & 0.049 & 0.825 & $10.923<0.001 * * *$ \\
\hline Convex hull area & 166940.000 & 2949.000 & 167086.000 & 2514.000 & 0.001 & 0.970 & $11.730<0.001 * * *$ \\
\hline Network density & 0.016 & 0.001 & 0.020 & 0.001 & 8.037 & $0.005 * *$ & $16.547<0.001 * * *$ \\
\hline No. of subnetworks & 163.000 & 3.382 & 158.000 & 3.236 & 0.009 & 0.924 & $6.3020 .012 *$ \\
\hline
\end{tabular}




\begin{tabular}{|c|c|c|c|c|c|c|c|c|}
\hline & \multicolumn{3}{|c|}{$\begin{array}{l}\text { Arenosols } \\
(n=3906)\end{array}$} & \multicolumn{3}{|c|}{$\begin{array}{l}\text { Cambisols } \\
(\mathrm{n}=3834)\end{array}$} & \multicolumn{2}{|l|}{ ANOVA } \\
\hline & Median & Lower Cl & Upper CI & Median & Lower $\mathrm{Cl}$ & Upper CI & $F$ & $p$ \\
\hline Number of nodes & 11.000 & 11.000 & 11.000 & 11.000 & 11.000 & 11.000 & $F(1,8.614)=1.542$ & 0.247 \\
\hline Number of links & 15.000 & 14.000 & 15.000 & 15.000 & 15.000 & 16.000 & $F(1,8.443)=3.793$ & 0.085 \\
\hline Mean node degree & 2.670 & 2.640 & 2.670 & 2.710 & 2.670 & 2.750 & $F(1,7.971)=9.239$ & $0.016 *$ \\
\hline Mean link length & 3.190 & 3.170 & 3.220 & 3.250 & 3.230 & 3.280 & $F(1,7.011)=11.322$ & $0.012 *$ \\
\hline SD link length & 1.620 & 1.600 & 1.640 & 1.670 & 1.640 & 1.690 & $F(1,8.334)=3.648$ & 0.091 \\
\hline Gamma index & 0.132 & 0.127 & 0.136 & 0.133 & 0.128 & 0.136 & $F(1,8.720)=0.355$ & 0.567 \\
\hline Beta index & 0.133 & 0.132 & 0.133 & 0.136 & 0.133 & 0.138 & $F(1,7.971)=9.239$ & $0.016 *$ \\
\hline Diameter & 19.000 & 18.600 & 19.500 & 19.300 & 18.800 & 19.700 & $F(1,7.957)=1.664$ & 0.233 \\
\hline Cost & 46.700 & 45.200 & 48.700 & 49.200 & 47.500 & 51.600 & $F(1,8.161)=4.717$ & 0.061 \\
\hline Global reach centrality & 0.058 & 0.057 & 0.059 & 0.058 & 0.057 & 0.059 & $F(1,7.645)=0.554$ & 0.479 \\
\hline Convex hull area & 63.500 & 59.800 & 67.000 & 62.500 & 58.500 & 66.000 & $F(1,8.132)=0.385$ & 0.552 \\
\hline Network density & 0.171 & 0.167 & 0.176 & 0.172 & 0.168 & 0.179 & $F(1,8.117)=0.013$ & 0.913 \\
\hline
\end{tabular}



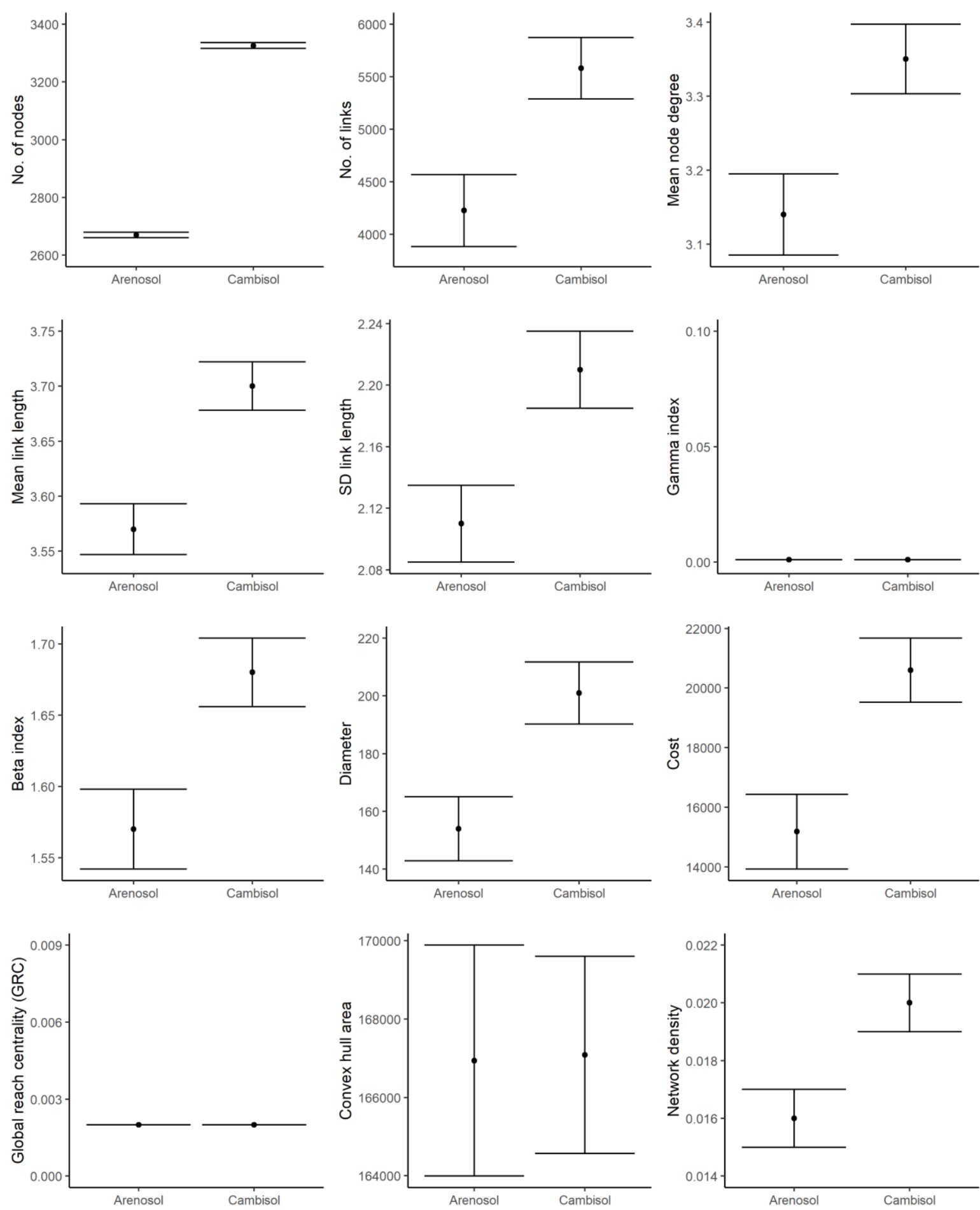

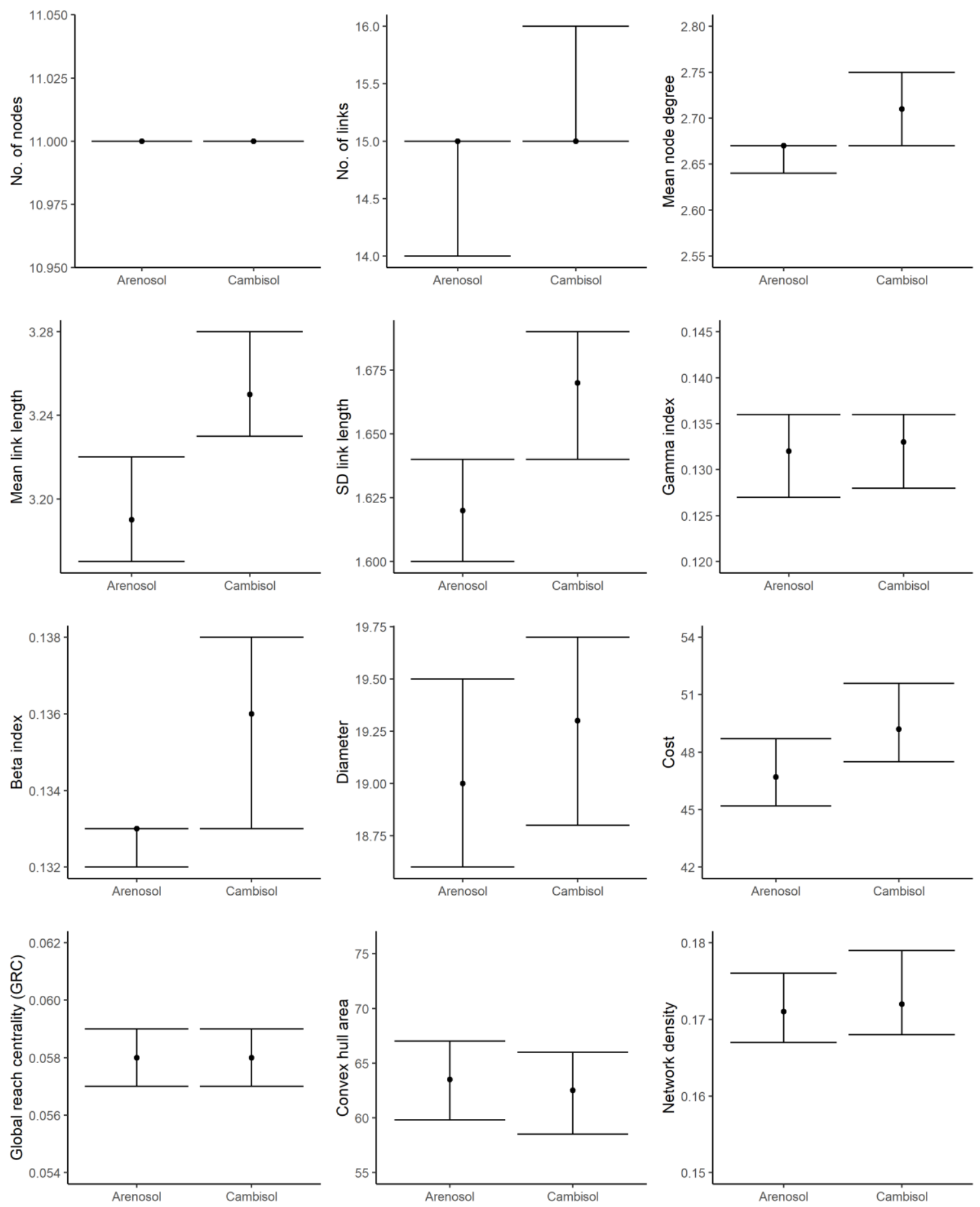

b.

355 Figure 1. The distribution of each network metric by soil type, for (a) main soil networks and (b) subnetworks. The point and error bars in (a) represent the estimated marginal mean and standard error for that network type and soil type, as determined by the ANOVAs (Table 4a), and the point and error bars in (b) represent the median and upper and lower $95 \%$ confidence intervals, respectively. Descriptions of the network metrics are in Table 1. 
At the main network level, the networks extracted from the Cambisols had significantly more nodes and links, a larger mean node degree and standard deviation of link length, and longer mean link length (Table 4a). These networks also had a higher beta index, higher cost, and higher density. While the main networks of the two soil types had significantly different gamma indexes, the absolute difference in the estimated marginal means between the two soil types was negligible $\left(<10^{-3}\right)$ (Table 4a, Fig 1a). At the subnetwork level, Cambisol networks had longer mean link length, and higher mean node degree and beta index (Table 4b). While not significant, Cambisol subnetworks also had noticeably larger number of links and standard deviation of link length, and higher cost (Fig 1b).

To control for the effect of replication on the significance, the profile ID was included in the ANOVAs as a mixed effect. This was significant for all metrics except mean and standard deviation of link length and diameter. Most profiles within each soil type at the main network level showed low absolute variation across the networks extracted from each however, and noticeably higher metric values for Cambisols than Arenosols (Fig A7a). At the subnetwork level, the distributions were quite similar across all profiles, but the Cambisol profiles showed more frequent and higher outliers.

\subsection{Agent-based model}

The ABM results showed significant differences across the different combinations of parameterisations and soil types, summarised in Tables 5 and 6 and Fig 2 . The simulations run on the Cambisol networks had significantly higher final population sizes (Tables 5 and 6, Fig 2b), and interactions between soil type and consumer and resource parameterisation were significant for several outcome variables (Table 6).

Table 5. The medians, first and third quantiles for agent-based model (ABM) outcome variable values across the two soil types. These values represent the overall results across all consumer and resource parameterisations. Descriptions of the variables are in Table 3.

\begin{tabular}{lrrrrrr} 
& \multicolumn{3}{c}{ Arenosols $(\mathbf{n}=\mathbf{3 7 5})$} & \multicolumn{3}{c}{ Cambisols $(\mathbf{n}=\mathbf{3 7 5})$} \\
\cline { 5 - 7 } & \multicolumn{1}{c}{ Median } & $\mathbf{1}^{\text {st }}$ Quantile & $\mathbf{3}^{\text {rd }}$ Quantile & \multicolumn{1}{c}{ Median } & 1 $^{\text {st }}$ Quantile & 3 $^{\text {rd }}$ Quantile \\
\hline Mean resource stock & 69.970 & 47.086 & 71.391 & 69.940 & 47.077 & 71.397 \\
SD resource stock & 33.266 & 22.262 & 34.123 & 33.300 & 22.276 & 34.112 \\
Entropy resource stock & 0.956 & 0.955 & 0.958 & 0.957 & 0.956 & 0.958 \\
Gini resource stock & 0.271 & 0.268 & 0.273 & 0.271 & 0.268 & 0.274 \\
Final population size & 4049.728 & 1967.921 & 5102.043 & 4890.158 & 2279.1560 & 5842.834 \\
\hline
\end{tabular}



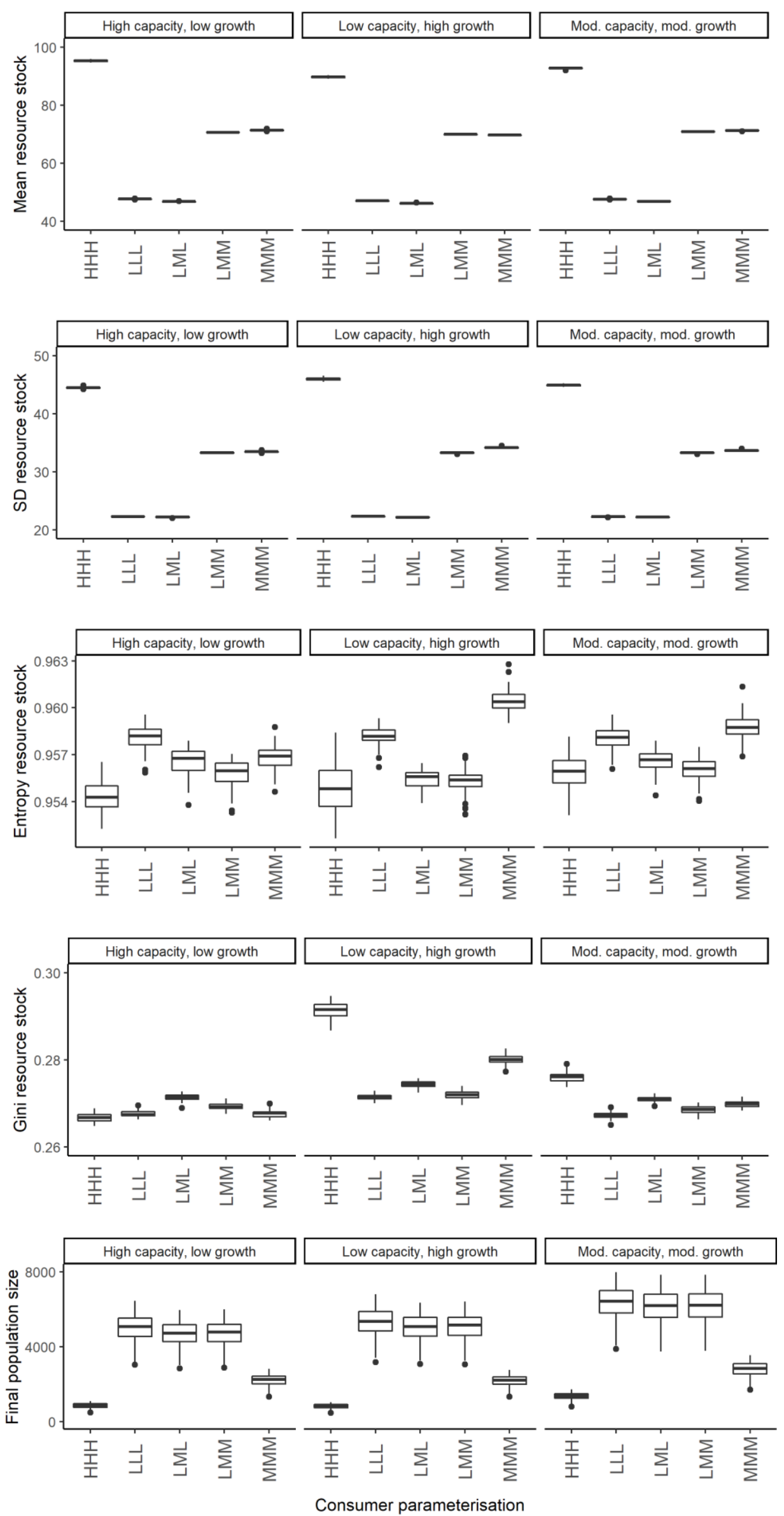

a. 

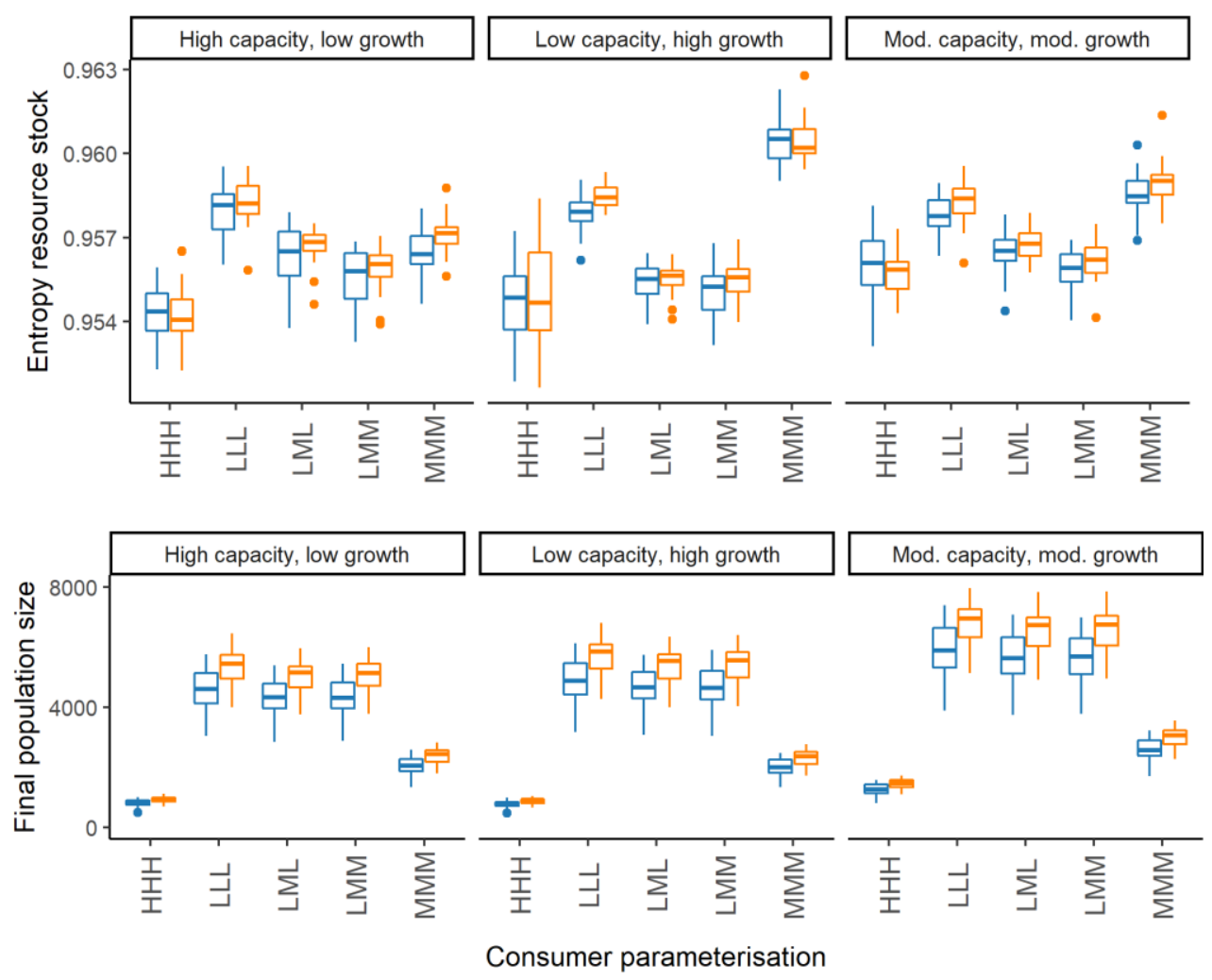

b.

Soil type 官 Arenosol 官 Cambisol

388 Figure 2. Distributions of (a) each agent-based model (ABM) outcome variable, grouped by resource parameterisation (columns, labelled at top) and consumer parameterisation ( $x$ axis within columns), across both soil types, and (b) ABM outcome variables that were significantly affected by soil type (represented by colour), grouped by resource parameterisation (columns) and consumer parameterisation ( $x$ axis within columns). The three-letter consumer parameterisation codes refer to the metabolism, consumption rate, and spawning threshold, respectively, where $H$ is high, $M$ is medium, and $L$ is low. Descriptions of the resource and consumer parameterisations are in Table 2, and descriptions of the outcome variables are in Table 3.

The ART ANOVAs showed that measured outcomes all differed significantly across consumer parameterisation, resource parameterisation, and consumer-resource parameterisation interactions. Final population size differed significantly by soil type, soil type-resource parameterisation interaction, and soil type-consumer parameterisation interaction. Mean resource stock also differed 
Table 6. Overview of Aligned Ranks Transformation ANOVA models of consumer population outcomes by consumer and resource parameterisation and soil type. The tests were Type III Wald F tests with Kenward-Roger degrees of freedom. Profile ID was included as a random effect. The asterisks designate level of significance: $p<0.1: \cdot p<0.05:{ }^{*}, p<0.01: * *, p<0.001:^{* * *}$. Descriptions of consumer and resource parameterisations are in Table 2 and descriptions of response variables are in Table 3.

\begin{tabular}{|c|c|c|c|}
\hline Response variable & Predictors & $\mathbf{F}$ & Significance \\
\hline & Consumer population & $F(4,710.063)=3585.924$ & $<0.001 * * *$ \\
\hline & Resource population & $F(2,710.063)=2585.400$ & $<0.001 * * *$ \\
\hline & Soil type & $F(1,9.692)=0.328$ & 0.560 \\
\hline & Consumer pop. $\mathrm{x}$ resource pop. & $F(8,710.094)=998.152$ & $<0.001 * * *$ \\
\hline & Consumer pop. $x$ soil type & $F(4,710.106)=1.460$ & 0.213 \\
\hline & Resource pop. $x$ soil type & $F(2,710.103)=3.513$ & $0.030 *$ \\
\hline \multirow{8}{*}{$\begin{array}{l}\text { Mean consumer } \\
\text { resource stock }\end{array}$} & Consumer pop. $x$ resource pop. $x$ & & \\
\hline & soil type & $F(8,710.102)=1.020$ & 0.419 \\
\hline & Consumer population & $F(4,710.185)=3629.337$ & $<0.001 * * *$ \\
\hline & Resource population & $F(2,710.292)=1137.215$ & $<0.001 * * *$ \\
\hline & Soil type & $F(1,9.212)=0.555$ & 0.475 \\
\hline & Consumer pop. $\mathrm{x}$ resource pop. & $F(8,710.233)=677.837$ & $<0.001 * * *$ \\
\hline & Consumer pop. $x$ soil type & $F(4,710.315)=2.164$ & 0.071 \\
\hline & Resource pop. $x$ soil type & $F(2,710.32)=0.538$ & 0.584 \\
\hline \multirow{8}{*}{$\begin{array}{l}\text { SD consumer } \\
\text { resource stock }\end{array}$} & Consumer pop. $x$ resource pop. $x$ & & \\
\hline & soil type & $F(8,710.306)=1.123$ & 0.345 \\
\hline & Consumer population & $F(4,710.030)=586.700$ & $<0.001 * * *$ \\
\hline & Resource population & $F(2,710.036)=59.661$ & $<0.001 * * *$ \\
\hline & Soil type & $F(1,9.861)=3.105$ & 0.109 \\
\hline & Consumer pop. $\mathrm{x}$ resource pop. & $F(8,710.025)=67.989$ & $<0.001 * * *$ \\
\hline & Consumer pop. $x$ soil type & $F(4,710.037)=2.364$ & 0.052 \\
\hline & Resource pop. $x$ soil type & $F(2,710.037)=0.241$ & 0.786 \\
\hline \multirow{8}{*}{$\begin{array}{l}\text { Entropy consumer } \\
\text { resource stock }\end{array}$} & Consumer pop. $x$ resource pop. $x$ & & \\
\hline & soil type & $F(8,710.037)=0.949$ & 0.475 \\
\hline & Consumer population & $F(4,710.677)=1296.640$ & $<0.001 * * *$ \\
\hline & Resource population & $F(2,711.086)=2004.095$ & $<0.001 * * *$ \\
\hline & Soil type & $F(1,7.791)=2.445$ & 0.158 \\
\hline & Consumer pop. $\mathrm{x}$ resource pop. & $F(8,710.847)=1005.251$ & $<0.001 * * *$ \\
\hline & Consumer pop. $\mathrm{x}$ soil type & $F(4,711.364)=0.470$ & 0.758 \\
\hline & Resource pop. $x$ soil type & $F(2,711.281)=2.502$ & 0.083 \\
\hline \multirow{9}{*}{$\begin{array}{l}\text { Gini consumer } \\
\text { resource stock }\end{array}$} & Consumer pop. $x$ resource pop. $x$ & & \\
\hline & soil type & $F(8,711.287)=0.614$ & 0.766 \\
\hline & Consumer population & $F(4,710.001)=1361.66$ & $<0.001 * * *$ \\
\hline & Resource population & $F(2,710.001)=604.376$ & $<0.001 * * *$ \\
\hline & Soil type & $F(1,9.998)=9.239$ & $0.012 *$ \\
\hline & Consumer pop. $\mathrm{x}$ resource pop. & $F(8,710.001)=33.651$ & $<0.001 * * *$ \\
\hline & Consumer pop. $\mathrm{x}$ soil type & $F(4,710.001)=41.516$ & $<0.001 * * *$ \\
\hline & Resource pop. $x$ soil type & $F(2,710.001)=5.039$ & $0.007^{* *}$ \\
\hline & Consumer pop. $x$ resource pop. $x$ & & \\
\hline Final population size & soil type & $F(8,710.001)=0.282$ & 0.972 \\
\hline
\end{tabular}




\section{6}

The entropy of consumer resource stocks and the final population size both showed considerable variation in the initial boxplots that was not explained by the consumer and resource parameterisation (Fig 2a), and the ANOVA results suggested that soil type was influential on final population size. Therefore, these were further explored with Kruskal-Wallis tests, first with profile ID as the grouping variable, then soil type (Table 7, also Fig 2b). Significant differences in profile ID were explored with Dunn post-hoc analysis. This showed that entropy differed significantly between profiles $\mathrm{D}$ and $\mathrm{H}$, which were Cambisol and Arenosol, respectively, while final population size differed significantly between several pairs of profiles, including both intra- and inter-type profile pairings.

\section{Table 7. Results of Kruskal-Wallis tests and Dunn post-hoc analysis comparing entropy of} consumer resource stocks and final population size by soil profile ID and soil type. The degrees of freedom for the Chi-square statistics were 11 and 1 for profile ID and soil type, respectively. Profile IDs A - G correspond to Cambisols, while profile IDs $\mathrm{H}-\mathrm{K}$ correspond to Arenosols. Significant pairs of profiles were identified at the level of $\alpha / 2$, where $\alpha=0.05$. Profile pairings in italics denote intertype pairs.

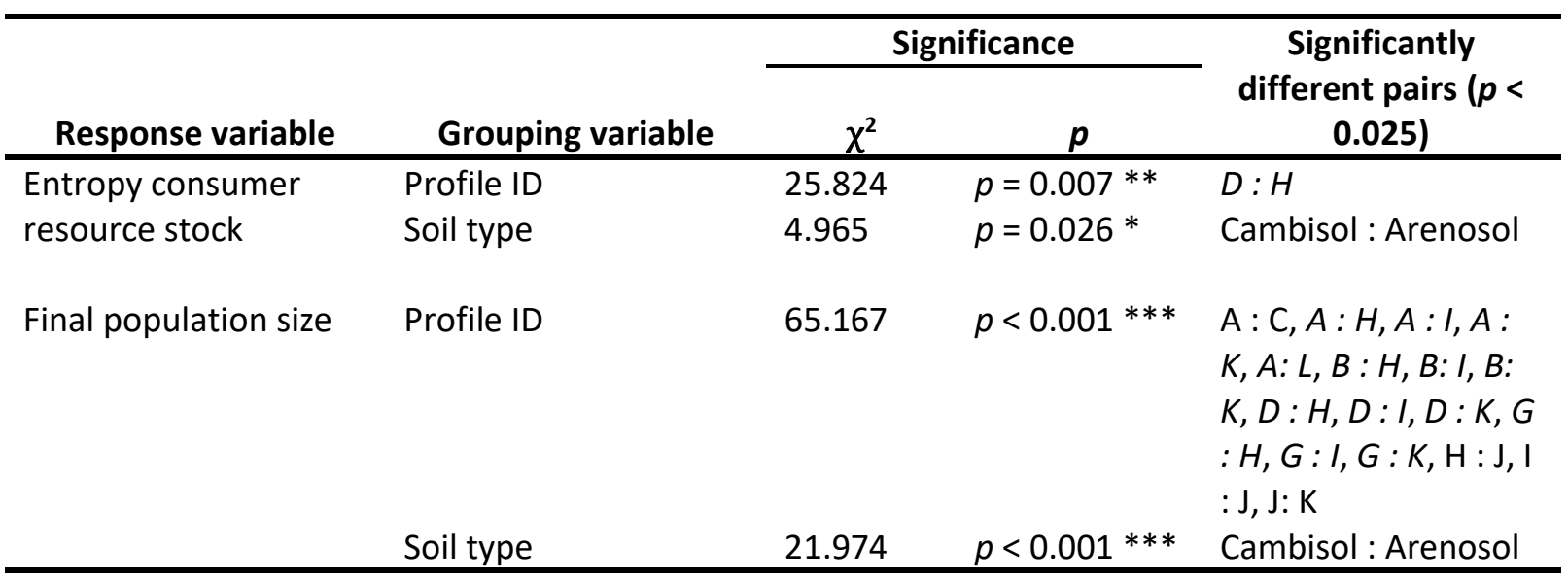




\subsection{Network analysis}

Given the known characteristics of the two soil types, the results of the network analysis suggest that the methodology developed here captures overall trends of soil structural development. Cambisols typically have more soil structure, higher porosity, higher levels of biotic activity, and greater stability than Arenosols (FAO, 2015). Correspondingly, the abstracted Cambisol soil networks analysed here showed higher values for the metrics measuring size, structure, and connectivity than the abstracted Arenosol soil networks did.

Specifically, the Cambisol soil networks had significantly more nodes and links, longer mean and standard deviation of link lengths, and higher total cost, density, and diameter (Table 4). This suggests more pore-creating activities modifying the soil, and a soil structural matrix that can support longer pores. This would also lead to higher water holding capacity, and increased internal drainage, both of which are commonly associated with Cambisols (FAO, 2015). In contrast, the smaller and less connected Arenosol networks have a low water-holding capacity, and the weaker coherence of their matrix material prevents longer pores from being stable, making them prone to erosion (FAO, 2015). Cambisols are also classified as more structurally developed than Arenosols, and contain more organic matter (FAO, 2015), both of which further validate the increased structure seen in the Cambisol networks here.

The global reach centrality, gamma index, and convex hull area were not as clearly differentiated between the Cambisol and Arenosol soil networks, however. The global reach centrality values were small and functionally identical, with an estimated marginal mean of 0.001 and 0.058 for both soil types at the main and subnetwork level, respectively (Table 4). Similarly, the estimated marginal mean gamma index for main networks of both soil types was 0.001 . This is likely due to the presence of a similar number of disconnected subnetworks within each soil network, limiting the total number of nodes that any given node can reach. The Cambisol main networks also had a slightly smaller range of convex hull areas, although the opposite trend emerges at the subnetwork level (Fig 1, Table 4). When this is decomposed by profile, the Cambisols show more variation and outliers across and within profiles for several metrics, including convex hull area (Fig A7), suggesting that soil type includes a greater heterogeneity of network sizes and structures. As with the other metrics, further work is required to establish ranges across different soil types and geographical regions, and to compare these metrics with those more commonly used in soil analysis. Overall, however, the differences between the Cambisols and Arenosols as captured in this analysis broadly reflects those expected, given the known differences in their properties.

The improved profile development and heterogeneity of Cambisols highlights their potential for agriculture and forestry, and in underpinning the diversity of a range of ecosystems. It is vital to manage them in a way that preserves and enhances their soil structure, however, to maintain their porosity and biodiversity, and resulting stability, drainage, and aeration. Similarly, Arenosols should be managed in a way that minimises their propensity for erosion and soil loss. In both cases, this can be accomplished through limiting or eliminating tillage (e.g. Young and Ritz, 2000; Helgason, Walley and Germida, 2010; Kravchenko et al., 2011), and increasing cover crops and native species (e.g. Fernández et al., 2019; Kravchenko et al., 2011). These provide additional organic inputs to the soil to promote an active and diverse soil biota, and therefore the positive feedback between biota, and structural development and stability (e.g. Oades, 1993; Young and Ritz, 2009; Crawford et al., 2012). The feasibility of the measurement and analysis methods presented here could provide a basis for estimating changes in structure over time and under different management strategies or 
environmental changes. This would help inform actions taken to preserve or improve the soil structure. However, further work is required to standardise the approach and demonstrate its application over multiple soil types.

As introduced in the Methods, the networks analysed here represent abstractions of the true soil structure present in the samples. This simplification is reasonable for analysing overall structural characteristics and heterogeneity and made the computation of the network metrics feasible. Although the short link lengths (Table 4) suggest that using Euclidean distance is likely negligibly different than measuring the path through the pixels, it does limit the interpretation of the findings we present. Specifically, the absolute values of the metrics cannot be taken to characterise the precise soil structure, but rather suggest general trends in structural development. As the exact size and shape of the pores was not preserved, many of the finer distinctions between networks may also be lost. This could cause the magnitudes of differences found between soil samples here to appear lower than they are. As discussed above, the relatively rapid, low-cost, and lightweight approach used here for estimating soil structure should be compared against more established approaches and metrics to determine its effectiveness. This methodology provides simplified and potentially inaccurate measurements of soil structure, but with further improvement it could be a suitable approach for rapid assessment of soil structure in the field. The results presented suggest that the methodology can still capture general known trends of heterogeneity within soil networks, meriting further refinements and application.

\subsection{ABM analysis}

The ABM evaluated the effects of and interactions between consumer and resource characteristics, and the structure of the abstracted soil networks, on the measured consumer outcomes. Overall, the results showed that the size and energetic heterogeneity of the consumer population was heavily influenced by the parameterisation of the consumer population and resource base, and their interactions. Moreover, while outcome variables were less directly affected by soil network structure, they were more influenced by the interactions between this network structure and consumer or resource parameterisations.

Across all simulations, measured outcomes varied most strongly across consumer and resource characteristics, and their combinations as overall consumer and resource parameterisations or types (Fig 2a, Table 6). Specifically, the mean, standard deviation, and entropy of consumer resource stocks, as well as the final population size, were most different across consumer types. These differences in outcome variables resulted from how each consumer population responded to the provided resource base. For example, the consumer populations with low metabolisms, low consumption rate, and a low energy requirement for spawning had a lower mean resource stock, and a higher final population size, for any given resource base. The consumers with high metabolisms, high consumption rate, and a high energy requirement for spawning had a lower final population size, but higher mean resource stock. This is similar to the distinction between $r$ strategists and K-strategists. In these simulations, the threshold for spawning and the active and basal metabolic rates appeared to have the largest impact on the measured outcome variables (Fig 2a). This is likely due to these parameters balancing one another in determining energy allocation between maintenance and reproduction (e.g. Brown et al., 2004; Kooijman, 2009).

In addition to consumer and resource characteristics, the soil type, and therefore soil network structure, also affected population size and diversity (Table 6). Specifically, the mean consumer resource stock and final population size showed significant differences across resource and soil type interactions, and final population size also showed significant differences between soil types (Table 
519 5). While the final population size and entropy also differed significantly across profiles (Table 6), post-hoc analysis revealed that for entropy this was only significant for inter-type profile pairings, and a slight difference was visible between groups when plotted (Fig 2b). This entropy is also known as the Shannon Index or Shannon-Wiener Index, and here measures the diversity or 'evenness' of the distribution of consumer resource stocks (Hill, 1973; Spellerberg and Fedor, 2003). Higher entropy therefore meant that given quantities of resource stock were represented in equal proportional abundance across the population. This is typically caused by groups of consumers emerging, where group members each have the same quantity of resource stock, but these quantities differ among groups. Over time, adaptations in this context could drive the system toward speciation. In these simulations, the larger populations supported by the larger Cambisol soil networks were more likely to have higher entropy, through different quantities of consumer resource stocks represented with equal proportional abundance.

\section{1}

The relatively low Gini coefficients (Table 5, Fig 2a) can also suggest the emergence of distinct groups of consumers with equal resource stocks, with similar numbers of consumers across the groups. As the Gini coefficient measures relative inequality, both inequality in resource stocks across groups, and more groups, cause it to increase. Equal group sizes can somewhat counter this. In both soil types however, as the consumers in a given simulation had identical characteristics, it is reasonable that they would have similar outcomes, slightly differing based on the subnetwork in which they found themselves, and the resource base available to them there. The similarity among subnetworks of the two soil types (Table $4 \mathrm{~b}$ ) suggests that the heterogeneity between soil types is more apparent at the main network level. As the consumers in these simulations were unable to move between subnetworks, they likely did not experience the full range of environmental heterogeneity between the soil types, which would have limited its effect on the measured outcomes.

Overall, the simulations highlight the differences in population size and diversity across consumer and resource parameterisations and interactions, soil and resource type interactions, and to a lesser extent, soil type on its own. Spatial heterogeneity, through both resource and network structural heterogeneity, can increase the microhabitat diversity (Anderson, 1978; Giller, 1996; Ettema and Wardle, 2002; Nielson et al., 2010), which was shown here through the increased evenness of consumer groups with different resource stocks. Similarly, the heterogeneous habitat of soils can limit competitive exclusion by providing structural and resource niches for different species (Bardgett, Yeates and Anderson, 2009), such that more structurally heterogeneous Cambisols have larger and more diverse populations (FAO, 2015). This was reproduced by the larger populations that emerged in the Cambisol simulations here, although speciation was not explicitly modelled. As with the findings of the network analysis, this emphasises the importance of preserving soil structure and providing adequate substrate for maintaining an active soil biota (e.g. Young and Ritz, 2009; Crawford et al., 2012; Fernández et al., 2019).

While the parameterisations presented here were limited, they revealed interesting effects of consumer and resource characteristics and interactions. The programming of the model itself, however, may also have had an impact on the outcome of consumer populations. For example, consumers moved randomly among resources rather than following any sort of search strategy, and there was no energetic penalty imposed for turning, which are simplifying assumptions based on the limited sensory and processing capabilities of most soil biota. This eliminated free parameters that would have to be tuned and analysed or sourced from limited data about specific soil biota metabolism and cognition. It also eliminated any effect that tortuosity of the network would have on consumer resource stocks, though. This may not be a correct assumption if turning has a higher 
burden physically, cognitively, or both. Furthermore, as consumers were not able to extend the network or move between subnetworks, they were unlikely to experience the full difference between soil networks, as discussed above. This may have led to a smaller effect of soil type on measured consumer outcomes.

Additionally, the extraction and simplification process used to create the soil networks may have affected the outcomes of the ABM. As the details of pore size and shape were not maintained, the consumers' ability to forage or hide in crevices was not intended to mimic the true range of consumer sizes and behaviours. Since predation was not included in the model, however, we did not intend to explore the hypothesised effect of physical niches on populations by limiting competitive exclusion and predation. While this would be an interesting future extension, and these changes could increase the observed effect of the soil network structure on consumer population outcomes, it would require refining the network extraction process as discussed above, as well as estimating ranges of consumer sizes and predation dynamics. The model presented here instead focussed on exploring the overall trends that might emerge in a population of consumers, rather than attempting to predict how specific populations might evolve. While its design limits the precision of the implications, it maintains the level of realism and generality assumed within the overall methodology (Levins, 1966).

\section{Conclusion}

This work has explored how analysing abstracted soil networks using standard network metrics, combined with simulations, can quantify the underlying structural and functional differences between soil types. We showed that networks derived from a brown forest soil, or Cambisol, were significantly larger, more connected, and more spatially heterogeneous than the networks derived from a less developed sandy beach soil, or Arenosol. These larger and more structured networks were in turn able to support larger populations of simulated consumers in an agent-based model $(A B M)$. The $A B M$ also demonstrated how the size and heterogeneity of the simulated population were significantly different across consumer and resource parameterisations, and interactions between these parameterisations and soil type.

In conclusion, standard network metrics applied to images can be a useful way to quickly assess the structure of networks within a soil profile, by capturing the broad structural differences between distinct soil types, in a way that can suggest functional differences as well. These initial estimates can be used on their own to survey an area more extensively or affordably, or coupled with more intensive analyses, such as three-dimensional imaging techniques. Agent-based modelling can also be used, when seeded with networks obtained from images or scans, to evaluate interactions between consumer and resource characteristics and network structure, and to quantify the impact these and other environmental factors have on the outcomes of simulated populations. Overall, combining network analysis and simulation modelling can provide unique insights on the structure, function, and diversity of an area of soil, and provide avenues for exploring the impact of future management, structural, or environmental changes. 


\section{Acknowledgements}

607 This work was supported by a joint PhD studentship sponsored by Lancaster University and the 608 James Hutton Institute, awarded to ND, and by the Rural and Environment Science and Analytical 609 Services (RESAS) Work Package 1.1 Soils. The authors gratefully acknowledge statistical advice from 610 Dr Vicki Davis, and constructive comments from Dr Jonathan Ball, Dr Uta Berger, and an anonymous 611 reviewer.

612

\section{Competing interests}

614 The authors declare no competing interests. 
617 Aitkenhead, M. J. et al. (1999) 'Modelling water release and absorption in soils using cellular

618 automata', Journal of Hydrology 220(1-2), pp. 104-112. doi: 10.1016/S0022-1694(99)00067-0.

619 Aitkenhead, M. et al. (2016) 'Automated soil physical parameter assessment using smartphone and 620 digital camera imagery', Journal of Imaging, 2(4). doi: 10.3390/jimaging2040035.

621

622

623

624

625

626

627

628

629

630

631

632

633

634

635

636

637

638

639

640

641

642

643

644

645

646

647

648

649

650

651

652

653

654

655

656

Anderson, J. M. (1978) 'Inter- and intra-habitat relationships between woodland cryptostigmata species diversity and the diversity of soil and litter microhabitats', Oecologia, 32, pp. 341-348. doi: https://doi.org/10.1007/BF00345112.

Baer, S. G. et al. (2005) 'Soil Heterogeneity Effects on Tallgrass Prairie Community Heterogeneity: An Application of Ecological Theory to Restoration Ecology', Restoration Ecology, 13(2), pp. 413-424. doi: 10.1111/j.1526-100x.2005.00051.x.

Barabási, A.-L. (2016) Network Science. Cambridge, UK: Cambridge University Press.

Bardgett, R. D., Yeates, G. W. and Anderson, J. M. (2009) 'Patterns and determinants of soil biological diversity', in Biological Diversity and Function in Soils, pp. 100-119.

Bivand, R. S., Pebesma, E. and Gomez-Rubio, V. (2013) Applied spatial data analysis with R. 2nd edn. New York, New York, USA: Springer. Available at: http://www.asdar-book.org.

ten Broeke, G., van Voorn, G. and Ligtenberg, A. (2016) 'Which Sensitivity Analysis Method Should I Use for My Agent-Based Model?', Journal of Artificial Societies and Social Simulation, 19(1), pp. 135. doi: 10.18564/jasss.2857.

Brown, J. H. et al. (2004) 'Toward a metabolic theory of ecology', Ecology, 85(7), pp. 1771-1789. doi: 10.1890/03-9000.

Crawford, J. W. et al. (2012) 'Microbial diversity affects self-organization of the soil - Microbe system with consequences for function', Journal of the Royal Society Interface, 9(71), pp. 1302-1310. doi: 10.1098/rsif.2011.0679.

Crawford, J. W., Ritz, K. and Young, I. M. (1993) 'Quantification of fungal morphology, gaseous transport and microbial dynamics in soil: an integrated framework utilising fractal geometry', Geoderma, 56(1-4), pp. 157-172. doi: 10.1016/0016-7061(93)90107-V.

Csardi, G. and Nepusz, T. (2006) 'The igraph software package for complex network research', InterJournal, Complex Sy, p. 1695.

Davis, N. et al. (2020). 'Trajectories toward maximum power and inequality in resource distribution networks', PLOS ONE, 15(3), e0229956. doi: https://doi.org/ 10.1371/journal.pone.0229956.

Davis, N. and Polhill, J. G. (2020). 'Soil network simulation (Version 1.0.0)', Zenodo.

http://doi.org/10.5281/zenodo.4001622

Davis, N. (2020) 'Soil network analysis functions (Version 1.0.0).' Zenodo.

http://doi.org/10.5281/zenodo.4001702

Dinno, A. (2017) 'dunn.test: Dunn's Test of Multiple Comparisons Using Rank Sums.' R package version 1.3.5. https://CRAN.R-project.org/package=dunn.test

Epskamp, S. et al. (2012) 'qgraph: Network Visualizations of Relationships in Psychometric Data', Journal of Statistical Software, 48(4), pp. 1-18.

Ettema, C. H. and Wardle, D. A. (2002) 'Spatial soil ecology', Trends in Ecology and Evolution, 17(4), pp. 177-183. doi: 10.1016/S0169-5347(02)02496-5. 
FAO (2015) World reference base for soil resources 2014: International soil classification system for naming soils and creating legends for soil maps, World Soil Resources Reports No. 106. Rome, Italy: Food and Agriculture Organization of the United Nations. doi: 10.1017/S0014479706394902.

Fernández, R. et al. (2019) 'Pore morphology reveals interaction of biological and physical processes for structure formation in soils of the semiarid Argentinean Pampa', Soil and Tillage Research. Elsevier, 191(January), pp. 256-265. doi: 10.1016/j.still.2019.04.011.

García-Palacios, P. et al. (2012) 'Plant responses to soil heterogeneity and global environmental change', Journal of Ecology, 100(6), pp. 1303-1314. doi: 10.1111/j.1365-2745.2012.02014.x.

Gargiulo, L., Mele, G. and Terribile, F. (2013) 'Image analysis and soil micromorphology applied to study physical mechanisms of soil pore development: An experiment using iron oxides and calcium carbonate', Geoderma. Elsevier B.V., 197-198, pp. 151-160. doi: 10.1016/j.geoderma.2013.01.008.

668

Giller, Paul S. (1996) 'The diversity of soil communities, the 'poor man's tropical rainforest", Biodiversity and Conservation, 5, pp. 135-168. doi: https://doi.org/10.1007/BF00055827.

Grimm, V. et al. (2006) 'A standard protocol for describing individual-based and agent-based models', Ecological Modelling, 198(1-2), pp. 115-126. doi: 10.1016/j.ecolmodel.2006.04.023.

672 Grimm, V. et al. (2010) 'The ODD protocol: A review and first update', Ecological Modelling, 221(23), 673 pp. $2760-2768$.

674 Hartemink, A. E. and Minasny, B. (2014) 'Towards digital soil morphometrics', Geoderma. Elsevier 675 B.V., 230-231, pp. 305-317. doi: 10.1016/j.geoderma.2014.03.008.

Hausser, J. and Strimmer, K. (2014) 'entropy: Estimation of Entropy, Mutual Information and Related Quantities.' R package version 1.2.1. https://CRAN.R-project.org/package=entropy

Helgason, B. L., Walley, F. L. and Germida, J. J. (2010) 'No-till soil management increases microbial biomass and alters community profiles in soil aggregates', Applied Soil Ecology. Elsevier B.V., 46(3), pp. 390-397. doi: 10.1016/j.apsoil.2010.10.002.

Hill, M. O. (1973) 'Diversity and evenness: A unifying notation and its consequences', Ecology, 54(2), pp. 427-432.

Kay M., Wobbrock J. (2020) 'ARTool: Aligned Rank Transform for Nonparametric Factorial ANOVAs.' doi: $10.5281 /$ zenodo.594511

Kooijman, S. A. L. M. (2009) Dynamic Energy Budget Theory for Metabolic Organisation. 3rd edn.

687 Kravchenko, A. N. et al. (2011) 'Long-term Differences in Tillage and Land Use Affect Intra-aggregate 688 Pore Heterogeneity', Soil Science Society of America Journal, 75(5), pp. 1658-1666. doi: 689 10.2136/sssaj2011.0096.

690 Kravchenko, A. N. and Guber, A. K. (2017) 'Soil pores and their contributions to soil carbon 691 processes', Geoderma. Elsevier B.V., 287, pp. 31-39. doi: 10.1016/j.geoderma.2016.06.027.

692 Kuznetsova, A., Brockhoff, P. B., Christensen, R. H. B. (2017) 'ImerTest Package: Tests in Linear Mixed 693 Effects Models', Journal of Statistical Software, 82(13), pp. 1-26. doi: 10.18637/jss.v082.i13

694 Lenth, R. (2020). 'emmeans: Estimated Marginal Means, aka Least-Squares Means', R package 695 version 1.5.0. https://CRAN.R-project.org/package=emmeans

696 Levins, R. (1966) 'The strategy of model-building in population biology', American Scientist. Sigma Xi, 697 54(4), pp. 421-431. www.jstor.org/stable/27836590. 
Lorscheid, I., Heine, B.-O. and Meyer, M. (2012) 'Opening the "black box" of simulations: increased transparency and effective communication through the systematic design of experiments"', Computational and Mathematical Organization Theory. Springer US, 18(1), pp. 22-62. doi: 10.1007/s10588-011-9097-3.

Mangiafico, S. (2020) 'rcompanion: Functions to Support Extension Education Program Evaluation.' R package version 2.3.25. https://CRAN.R-project.org/package=rcompanion

Mones, E., Vicsek, L. and Vicsek, T. (2012) 'Hierarchy measure for complex networks', PLoS ONE, 7(3), pp. 1-10. doi: 10.1371/journal.pone.0033799.

Nielson, U. N. et al. (2010) 'The enigma of soil animal species diversity revisited: The role of smallscale heterogeneity', PLOS ONE, 5(7), e11567. doi: 10.1371/journal.pone.0011567.

Oades, J. M. (1993) 'The role of biology in the formation, stabilization and degradation of soil structure', Geoderma, 56(1-4), pp. 377-400. doi: 10.1016/0016-7061(93)90123-3.

Pebesma, E. J. and Bivand, R. S. (2005) 'Classes and methods for spatial data in R', R News, 5(2). Available at: https://cran.r-project.org/doc/Rnews/.

Polhill, G. and Gimona, A. (2014) 'Using genetic algorithms to fit species and habitat parameters for modelling the effect of climate change on species distributions with stochastic patch occupancy models', Proceedings - 7th International Congress on Environmental Modelling and Software: Bold Visions for Environmental Modeling, iEMSs 2014, 3.

R Core Team (2020) 'R: A Language and Environment for Statistical Computing', $R$ Foundation for Statistical Computing. Vienna, Austria, p. https://www.R-project.org. Available at: http://www.rproject.org.

Rockafellar, R. T. (1970) Convex Analysis. Princeton, NJ: Princeton University Press.

Rodrigue, J. P. (2017) The Geography of Transport Systems. Hofstra University, Department of Global Studies \& Geography. Available at: https://transportgeography.org.

Roshier, D.A., Doerr, V. A. J. and Doerr, E. D. D. (2008) 'Animal movement in dynamic landscapes: interaction between behavioural strategies and resource distributions', Oecologia, 156, pp. 465-477. doi: 10.1007/s00442-008-0987-0.

Spellerberg, I. F. and Fedor, P. J. (2003) 'A tribute to Claude Shannon (1916-2001) and a plea for more rigorous use of species richness, species diversity and the "Shannon-Wiener" Index', Global Ecology and Biogeography, 12, pp. 177-179. doi: 10.1046/j.1466-822X.2003.00015.x.

Stevens, R. D. and Tello, J. S. (2011) 'Diversity begets diversity: relative roles of structural and resource heterogeneity in determining rodent community structure', Journal of Mammalogy, 92(2), pp. 387-395. doi: 10.1644/10-mamm-a-117.1.

Sturges, H. A. (1926) 'The choice of a class interval', Journal of the American Statistical Association, 21(153), pp. 65-66. doi: 10.1080/01621459.1926.10502161.

Tews, J. et al. (2004) 'Animal species diversity driven by habitat heterogeneity/diversity: The importance of keystone structures', Journal of Biogeography, 31(1), pp. 79-92. doi: 10.1046/j.03050270.2003.00994.x.

Velde, B., Moreau, E. and Terribile, F. (1996) 'Pore networks in an Italian vertisol: Quantitative characterisation by two dimensional image analysis', Geoderma, 72(3-4), pp. 271-285. doi: 10.1016/0016-7061(96)00033-X.

Vezzani, F. M. et al. (2018) 'The importance of plants to development and maintenance of soil 
structure, microbial communities and ecosystem functions', Soil and Tillage Research. Elsevier,

741 175(March 2017), pp. 139-149. doi: 10.1016/j.still.2017.09.002.

742 Wickham, H. (2016) ggplot2: Elegant Graphics for Data Analysis. New York: Springer-Verlag.

743 Available at: http://ggplot2.org.

744 Wickham, H. et al. (2019) 'dplyr: A Grammar of Data Manipulation'.

745 Wijesinghe, D. K., John, E. A. and Hutchings, M. J. (2005) 'Does pattern of soil resource heterogeneity 746 determine plant community structure? An experimental investigation', Journal of Ecology, 93(1), pp.

747 99-112. doi: 10.1111/j.1365-2745.2004.00934.x.

748 Wobbrock, J., Findlater, L., Gergle, D., Higgins, J. (2011) 'The Aligned Rank Transform for

749 Nonparametric Factorial Analyses Using Only ANOVA Procedures.' In Proceedings of the ACM

750 Conference on Human Factors in Computing Systems (CHI '11)', pp. 143-146.

751 Young, I. M., Crawford, J. W. and Rappoldt, C. (2001) 'New methods and models for characterising

752 structural heterogeneity of soil', Soil and Tillage Research, 61(1-2), pp. 33-45. doi: 10.1016/S0167-

753 1987(01)00188-X.

754 Young, I. M. and Ritz, K. (2000) 'Tillage, habitat space and function of soil microbes', Soil and Tillage 755 Research, 53(3-4), pp. 201-213. doi: 10.1016/S0167-1987(99)00106-3.

756 Young, I. M. and Ritz, K. (2009) 'The habitat of soil microbes', in Biological Diversity and Function in 757 Soils, pp. 31-43.

758 Zeileis, A. (2014) 'ineq: Measuring Inequality, Concentration, and Poverty.' R package version 0.2-13. 


\section{Appendices}

762

763

Appendix 1. Network extraction process

764 For all images:

765

766

767

768

769

770

771

772

773

774

775

1. Convert the image to a text file containing RGB triplets

2. Identify and eliminate all non-soil pixels (set to -1)

3. Calculate mean pixel intensity at all points

4. Adjust pixel intensity to remove variations in brightness across image

5. Threshold the image to retain the darkest $30 \%$ soil pixels

6. Carry out erosion and thinning operators

7. Clean image to produce skeletal pixels

8. Identify networks

9. Remove redundant pathways

10. Calculate distances between nodes

11. Save the network 


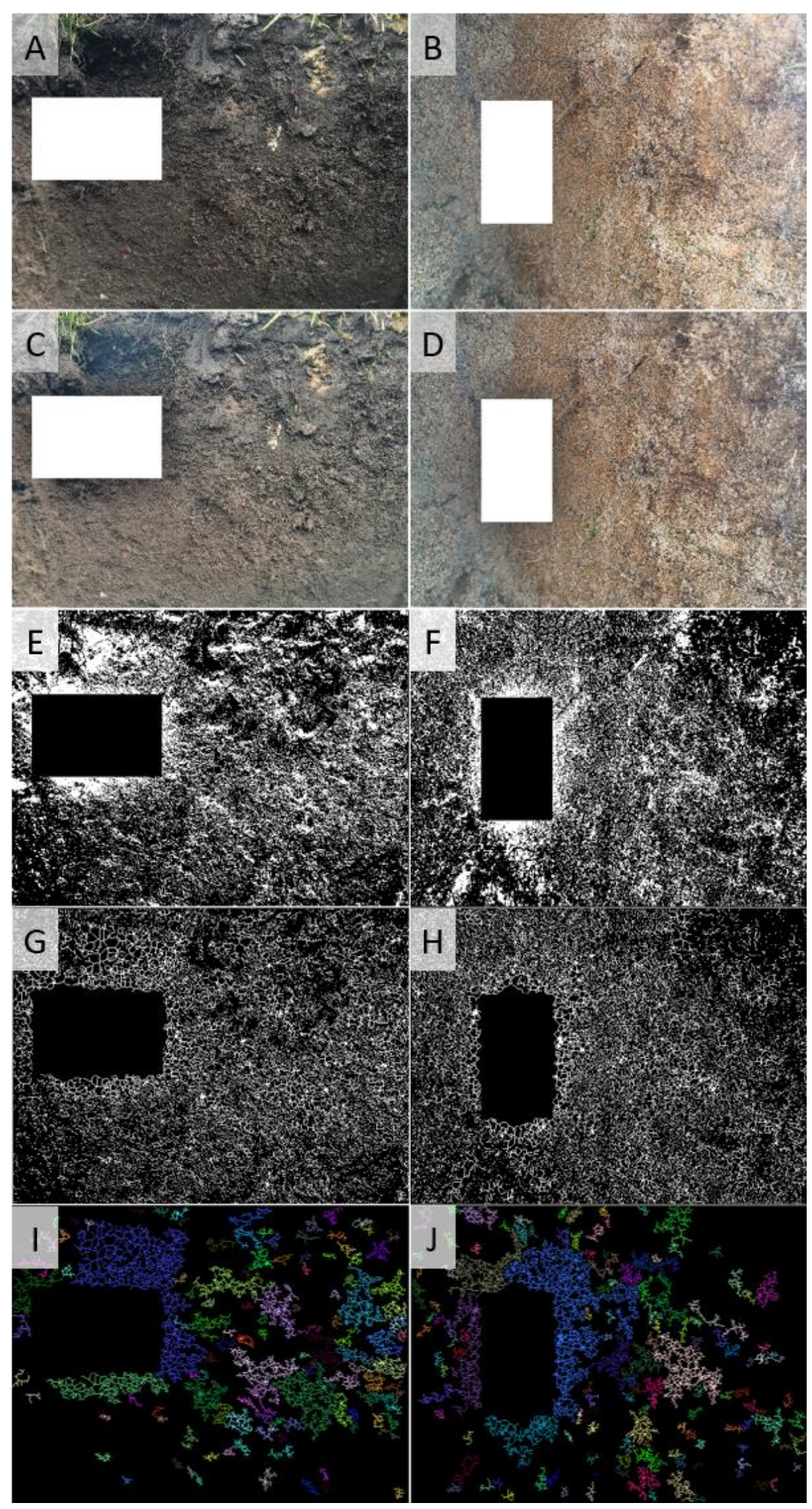

Figure A1. Soil image morphology process for a Cambisol (a) and Arenosol (b) profile image. Steps show include (c-d) colour correction, (e-f) thresholding, (g-h) erosion and thinning operations, and ( $\mathrm{i}-$

779 j) subnetwork identification. White areas represent colour correction cards, which were excised. 


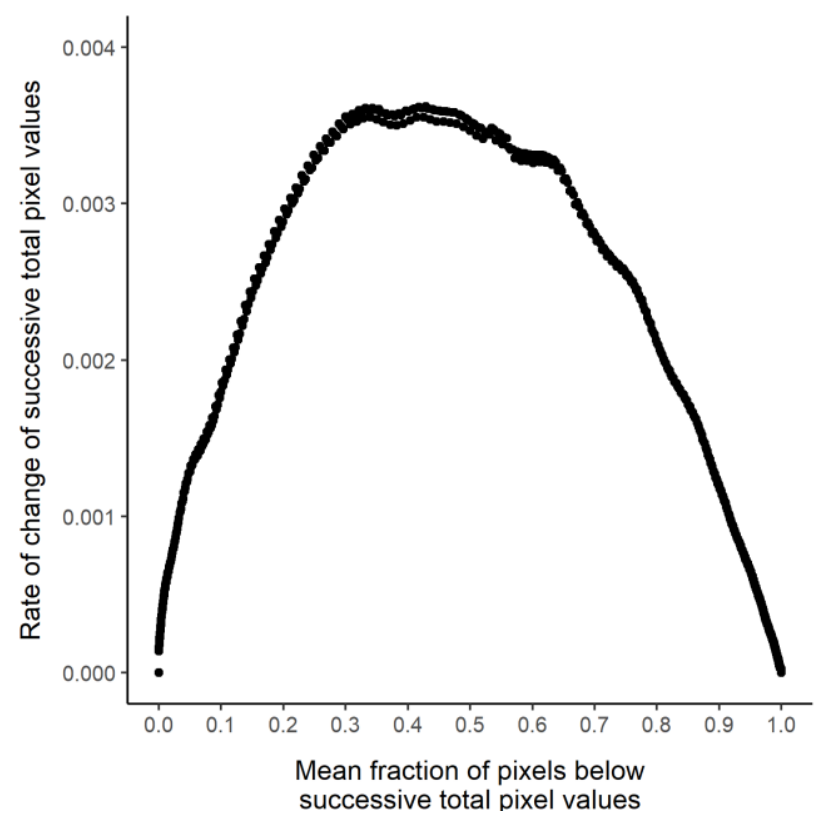

Figure A2. The rate of change of mean fraction of pixels for each mean fraction below a given

threshold value. The plot starts on the left with pixel values of 0 , with no pixels below this value, and ends on the right with pixel values of 755 (with correction card removed from image). The y-axis shows the rate of change of the mean fraction of pixels below each value.
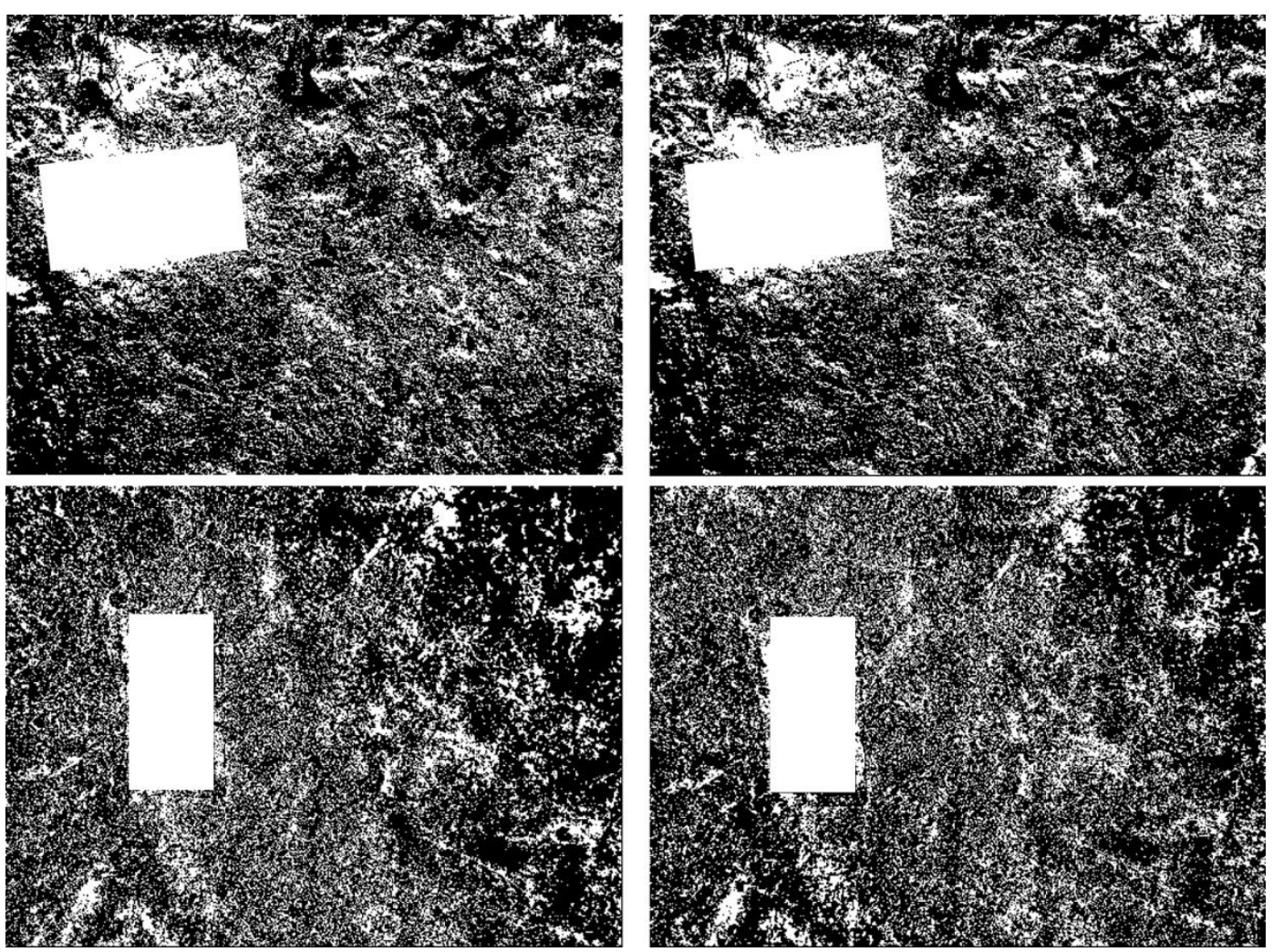

Figure A3. Comparison of different images from the same pit after thresholding. The two pairs of images from each pit are arranged horizontally. The white rectangle is the correction card. The thresholding process was the same as described in Section 2.2, where the darkest $30 \%$ of pixels have been retained as pores, and other pixels removed. 


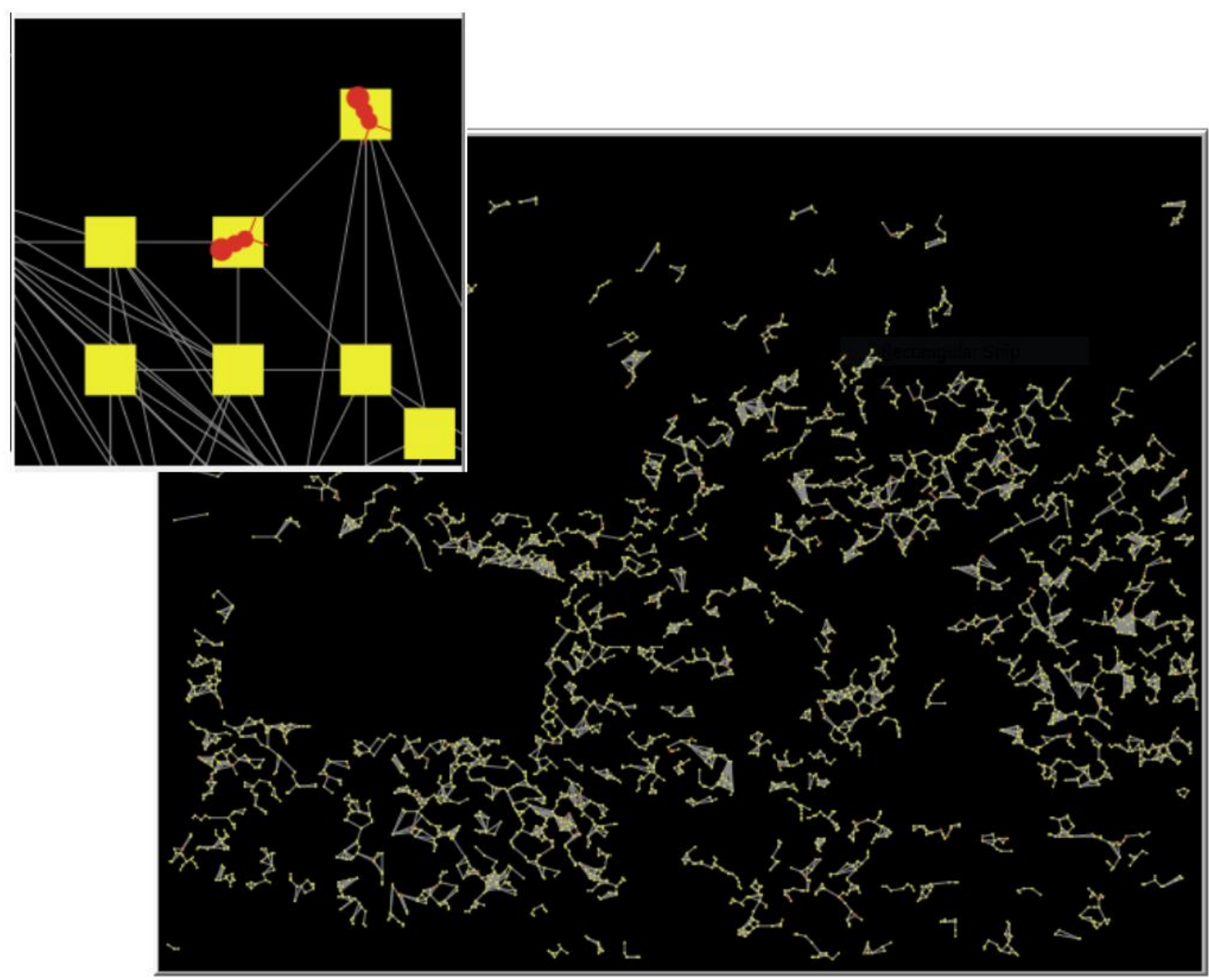

Figure A4. A screenshot of the model. The inset at the top left shows an enlarged version of some of the resource nodes (yellow squares) and agents (red ants).

I. Model purpose

a. The model is designed to be an analytical tool to explore the heterogeneity in resource supply potential of a network by populating it with idealised energyconsuming agents, and to quantify the effects of consumer, resource, and network characteristics on resulting consumer population outcomes.

II. Entities, state variables, and scales

a. Consumer entities

i. State variables

\begin{tabular}{ll}
\hline Property & Description \\
\hline Location & The resource on which the consumer is located \\
Target location & The resource to which the consumer will move next \\
Active? & Whether a consumer is active (or dead) \\
\hline
\end{tabular}




\begin{tabular}{|c|c|}
\hline Property & Description \\
\hline Basal metabolism & How much resource an agent needs per day to stay alive \\
\hline Active metabolism & How much resource an agent uses with each step \\
\hline Resource stock & How much resource an agent has consumed but not metabolised \\
\hline Consumption rate & $\begin{array}{l}\text { Maximum number of resource units that an agent takes from a resource it } \\
\text { visits, per timestep }\end{array}$ \\
\hline Spawn energy & $\begin{array}{l}\text { How much energy an agent requires to spawn (depletes this quantity from } \\
\text { stocks and passed to offspring as starting quota) }\end{array}$ \\
\hline
\end{tabular}

b. Resource entities

i. State variables

\begin{tabular}{ll}
\hline Property & Description \\
\hline Current supply & The current quantity of resource at this point \\
Resource capacity & How much energy is stored in a resource when it is full \\
Regrow rate & The amount the resource regrows each timestep \\
\hline
\end{tabular}

810

\begin{tabular}{ll}
\hline Property & Description \\
\hline Resource capacity & How much energy is stored in a resource when it is full \\
Regrow rate & The amount the resource regrows each timestep \\
\hline
\end{tabular}

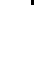

西

\section{c. Link entities}

\begin{tabular}{ll}
\hline Property & Description \\
\hline Length & The length of the link - determines energy and time required to traverse it
\end{tabular}

d. Scales

\begin{tabular}{ll}
\hline Property & Description \\
\hline Timestep & $\begin{array}{l}\text { A single unit of time in the model, defined as that which is required for } \\
\text { consumers to move } 1 \text { pixel (approximately } 0.3-0.5 \mathrm{~mm}), \text { and for which they } \\
\text { require basal-metabolism units of energy. }\end{array}$ \\
$\begin{array}{l}400 \times 500, \text { determined by the size of the soil networks used as the } \\
\text { environment. }\end{array}$ \\
\hline
\end{tabular}

a. Consumers start on random nodes around a pre-specified network, where nodes are resource patches.

b. Consumers move around the network randomly following links. If they find a resource patch, they consume as much as they can from it, and the patch depletes.

i. Consumers require basal-metabolism units of resource per timestep. If they do not consume this resource, they die. 
ii. Consumers can stay put on a resource and consume it (consumptionrate units consumed per timestep), but it depletes, and if there is no more resource there then they move on.

iii. Consumers metabolise active-metabolism units of resource per patch of link that they cross.

iv. If there is more than one agent on a resource patch, they each take consumption-rate units per timestep, or split the remainder if there is not enough resource remaining for them to each get consumption-rate units.

c. If consumers have twice as much energy as the set spawn-energy, they can spawn new consumers (who take the same amount of resource-stock from their parent that the parent started with, so now parent and offspring both have the same resource-stock).

d. Resources regrow at a constant rate (regrow-rate) per timestep, up to their maximum capacity (resource-capacity).

IV. Design concepts

a. Basic principles

i. Consumers attempt to consume as much free energy from a resource as they are able, to maximise energy reserves for future movement, and spawning capability.

ii. Conservation equations: energy and matter cannot be created (except at the start of the simulation) or destroyed. In spawning, this is represented by consumers transferring some of their energy to their offspring. Consumers only die when their energy reserves are completely depleted (starvation).

iii. Entropy production: some resource energy is consumed in movement and cannot be recaptured.

b. Emergence

i. The distribution of consumers in space around the network and the distribution of resource stocks across the consumers both emerge from the interactions in the model.

c. Objectives

i. The consumers' objective is to consume as much resource energy as possible, allowing them to stay alive, move, and potentially reproduce.

d. Prediction

i. Consumers do not 'predict' the results of their course of action per se, they are random walkers, but they do 'predict' that they will die if they stay in a non-resource patch, or depleted resource patch, so they keep moving.

e. Sensing

i. Consumers can sense if they are on a resource patch or not, and if it has any resource energy in it. They also know the link-neighbours of the resource patch that they are currently on.

f. Learning

i. Consumers are random walkers; they do not learn in any capacity.

g. Adaptation

i. The population adapts to fill the network in a way that reflects the density of resource availability in that area, as consumers will cluster and reproduce around resources where they can consume what they need. 
h. Interaction

i. Consumers interact stigmergically through their consumption of resources. While they do not interact directly in any meaningful way, their consumption of resources affects the availability of resources for others to consume.

i. Collectives

i. There are no collectives present.

j. Stochastic elements

i. Consumers are initialised in random locations and move randomly. Additionally, resources are all initialised with random maximum capacity between 1 and maximum-resource-capacity and regrow rates between 1 and maximum-regrow-rate.

k. Observation

i. Number of currently active ('alive') consumers at each timestep.

ii. Mean, standard deviation (SD), Gini coefficient, and entropy of the distribution of consumer resource stocks at each timestep.

iii. The resource capacity and regrow rate of each resource at the start of the simulation.

iv. The resource stock and location of each active consumer at 10, 100, 500, 1000 , and 2000 timesteps.

V. Initialisation

a. The network was supplied as two Comma-Separated Values (CSV) files: one of resource node locations and another of the connections between the resource nodes. The node locations and connections were determined during the process of extracting the soil network from a soil profile image, as described in the main text (Section 2.2). The resource and consumer types and parameters were specified in an Extended Markup Language (XML) file. The models were initialised with 500 consumers located on random resource nodes throughout the network. The consumers each began with 30 resource units in their resource-stock, and metabolic rates, consumption rate, and spawn energy thresholds as specified in the XML file. Resources were all initialised with random maximum capacity between 1 and maximum-resource-capacity and regrow rates between 1 and maximum-regrow-rate and began the simulation at full capacity.

VI. Input data

a. This model has no input data.

VII. Submodels

a. Regrowth of resources: at each timestep, all resources that are less than their maximum capacity regrow by regrow-rate units.

b. Consuming resources: at each timestep, all consumers currently located on a resource node check whether there is any resource available at that node. If there is enough for each consumer to take consumption-rate units, they do, and these are added to their resource-supply. If there is not enough, each consumer receives what is at the resource, divided by the number of consumers at the resource. If there is no resource available at that node, the consumer identifies a new target-node, selecting randomly from the other resources connected to the first, and moves to the target-node. 
c. Spawning new consumers: at each timestep, consumers check whether they have twice the amount of energy specified as spawn-energy in their resourcestock. If so, they spawn a new consumer who is an exact clone of themselves. The new consumer starts with spawn-energy units as their initial resourcesupply, and the parent consumer loses spawn-energy units of resource from their resource-stock.

d. Check consumer resource stocks: at each timestep, all consumers check whether they have more than resource-requirement units, or their basal metabolism, of resource in their resource-supply. If they do, they consume resourcerequirement units, removing them from their resource-supply, otherwise they die. 
931 First, a pre-test was conducted to determine the number of time steps for which to run the

932 simulations, and the number of replicates of each parameter set that were necessary for the outputs

933 to reach equilibrium (ten Broeke, van Voorn and Ligtenberg, 2016). The first set of 500 runs used

934 varied parameter values and a fixed network architecture, determined by Latin Hypercube Sampling

935 from the range of values for global analysis (Table A1). One replicate of each parameter set was run

936 for 3000 timesteps, and the output variables were plotted to determine whether the model reached

937 a stable state, and if so, when. As all runs showed stability in output parameters after $500-1000$

938 timesteps (Fig. A5), apart from small variations due to stochasticity, the final output variable values

939 for all future runs were calculated as the mean of the values at timesteps 500, 750, and 1000.

Table A1. Parameter ranges used for testing to determine length of simulations. Values shown are 942 the minimum and maximum for that parameter. Latin Hypercube Sampling was used to generate the 943 values, which were then multiplied by the range plus the minimum, to get the value for the 944 parameter for testing.

\begin{tabular}{lr}
\hline Parameter & Value \\
\hline Initial population size & 50,1000 \\
Consumer basal metabolism & 1,3 \\
Consumer active metabolism & 1,3 \\
Initial consumer resource stock & 20,50 \\
Consumer consumption rate & 5,10 \\
Consumer spawn energy & 50,100 \\
Maximum resource capacity & 20,50 \\
Maximum resource regrowth rate & 10,20 \\
\hline
\end{tabular}



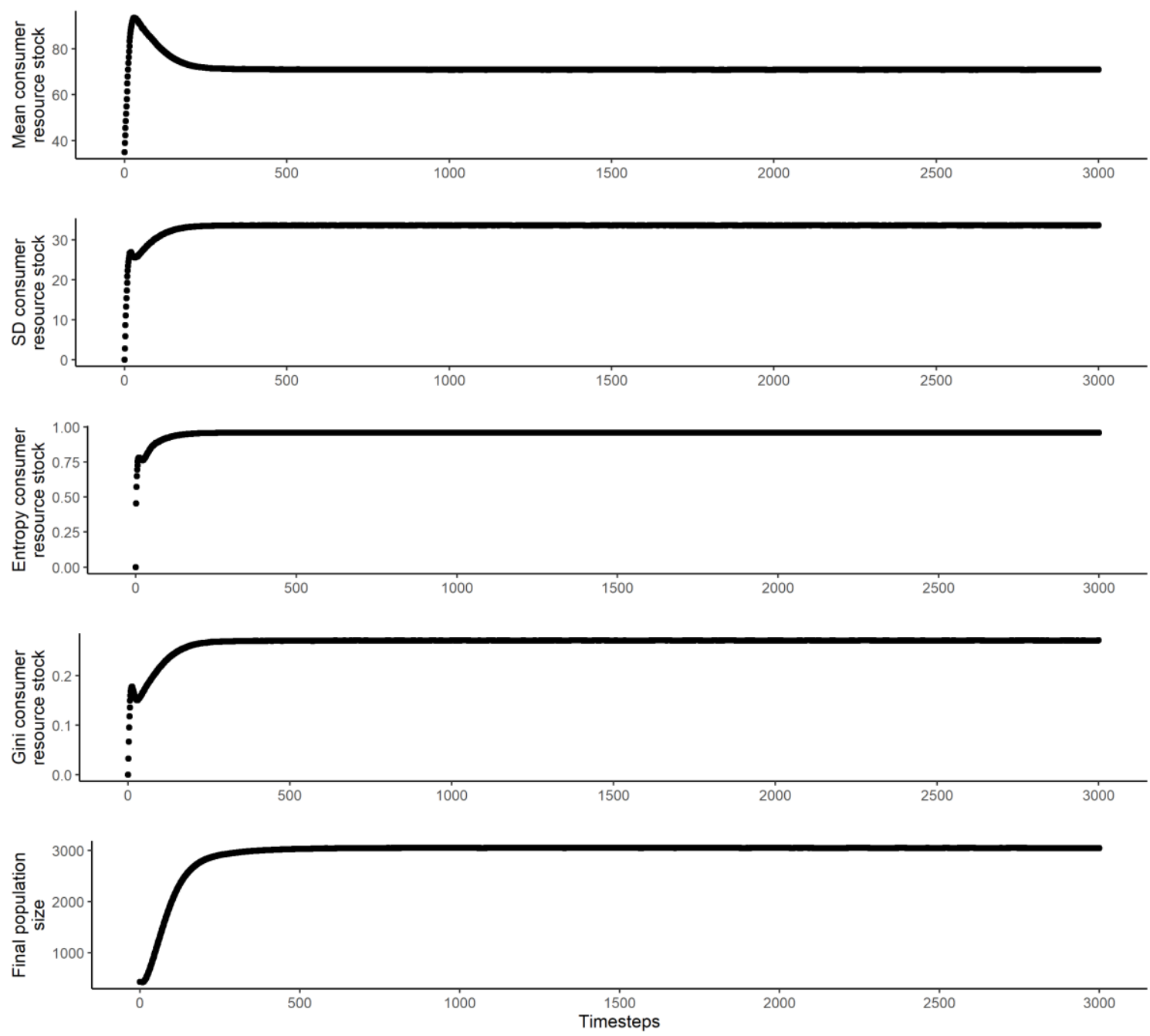

Figure A5. Stability plots from testing to determine the length of simulations. Shown are values

948 averaged for each timestep over 500 runs.

950 The second set of pre-test runs used the baseline parameter values for all parameters, and a fixed network, which we repeated 100 times. We then calculated a rolling coefficient of variation for the output variables, including progressively more replicates (Fig. A6). The coefficients of variation for all output variables stabilised around 10 runs. Plotting the distribution of the output variables at that point show approximate normality, such that the mean value across runs is a reasonable measure of centre. Therefore, for all future simulations, the mean of the outcome variables across 10 replicates was used to reduce the effects of stochasticity on the output. As the mean value across replicates was used, there was no effect from replication on the experimental results. 

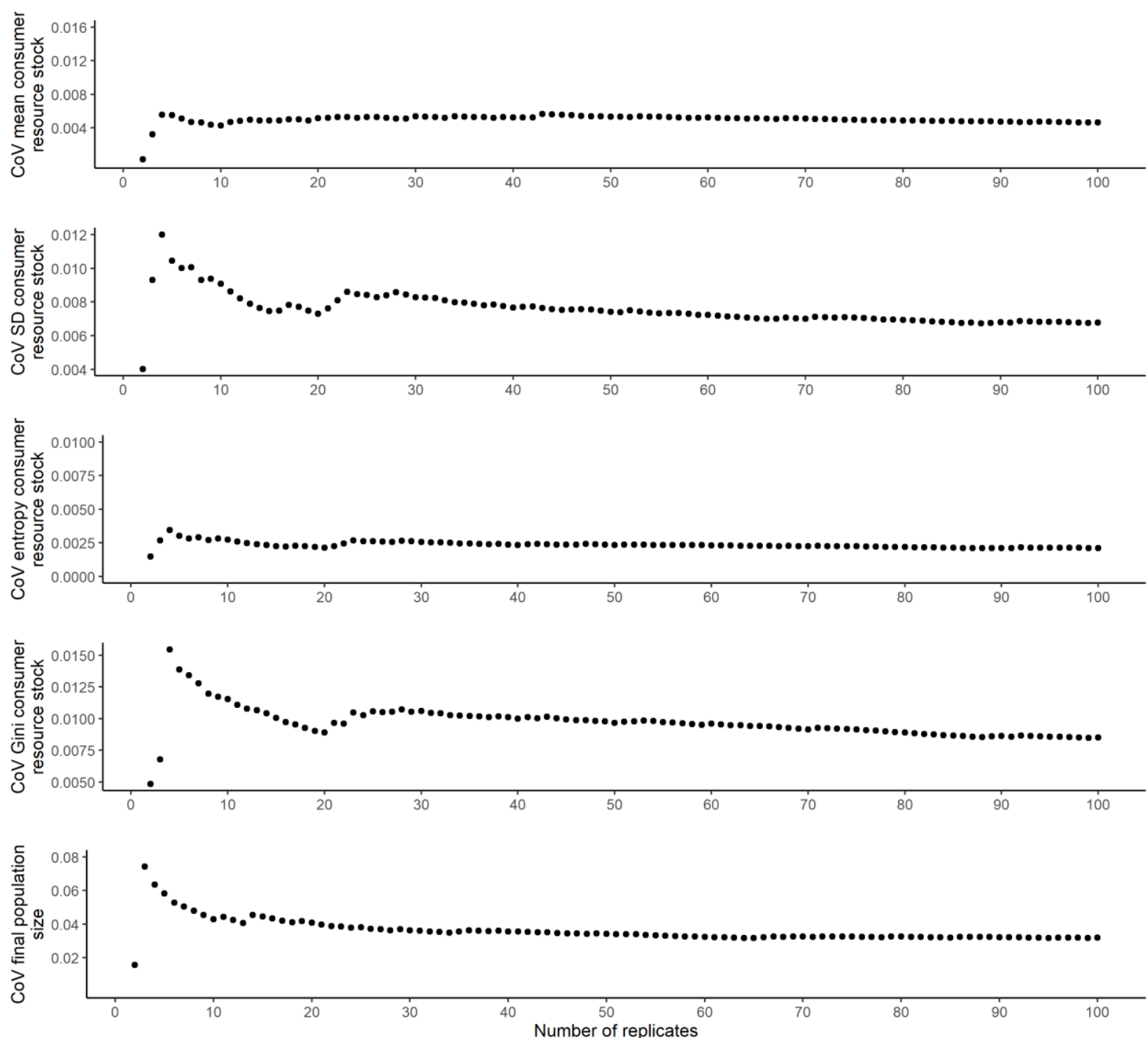

Figure A6. Plots of rolling Coefficient of Variation (CoV) for each outcome variable against the number of replicates included in its calculation. This was used to determine number of replicates needed to average across to minimise stochasticity in output variables.

After the pre-test, we used the One-Factor-at-a-Time methodology to identify which of the control variables significantly affected the output variables, and which could be held constant. For this test, the four control variables (initial consumer population, initial consumer resource stock, maximum resource regrowth rate, and maximum resource capacity) were varied across four levels each, changing one variable at a time, while holding all other variables constant at middle values for each. Both the maximum resource regrow rate and maximum resource capacity significantly affected the output variables, while initial consumer resource stock did not (Table A2). The initial consumer population size significantly affected all but the standard deviation of consumer resource stock (Table A2e), but as the magnitude of the effect was quite small, both the initial population size and initial consumer resource stock were held constant at middle values for the rest of the simulations. 
Table A2. Regression results from One-Factor-at-a-Time analysis. This was used to identify which control parameters could be fixed, and which significantly affected the outcome variables and needed to be explored. The asterisks designate level of significance: $p<0.1: \cdot p<0.05:{ }^{*}, p<0.01$ : $* *, p<0.001: * * *$.

a. Mean consumer resource stock

\begin{tabular}{lrcrl}
\hline & \multicolumn{3}{c}{ Standard } & \\
& Estimate & error & t value & $\boldsymbol{p}$ \\
\hline Intercept & 54.360 & 0.100 & 543.566 & $0.000^{* * *}$ \\
Initial population size & 0.000 & 0.000 & -3.728 & $0.002^{* * *}$ \\
Initial consumer resource stock & 0.000 & 0.001 & 0.100 & 0.920 \\
Maximum resource regrow rate & 0.054 & 0.004 & 12.874 & $0.000^{* * *}$ \\
Maximum resource capacity & 0.060 & 0.001 & 41.088 & $0.000^{* * *}$ \\
\hline $\mathrm{F}(4,251)=467(p<0.001)$ & $\mathrm{R}^{2}=0.88$ & & &
\end{tabular}

b. SD consumer resource stock

\begin{tabular}{lrrrl}
\hline & Estimate & Standard & & \\
& error & t value & \multicolumn{1}{c}{$\boldsymbol{p}$} \\
\hline Intercept & 27.110 & 0.038 & 711.283 & $0.000^{* * *}$ \\
Initial population size & 0.000 & 0.000 & 0.948 & 0.344 \\
Initial consumer resource stock & 0.000 & 0.000 & 0.333 & 0.739 \\
Maximum resource regrow rate & 0.016 & 0.002 & 10.073 & $0.000^{* * *}$ \\
Maximum resource capacity & -0.010 & 0.000 & -17.718 & $0.000^{* * *}$ \\
\hline $\mathrm{F}(4,251)=104.1(p<0.001)$ & $\mathrm{R}^{2}=0.62$ & & &
\end{tabular}

979

\section{c. Entropy consumer resource stock}

\begin{tabular}{lrrrl}
\hline & \multicolumn{3}{c}{ Standard } \\
& Estimate & error & t value & \multicolumn{1}{c}{$\boldsymbol{p}$} \\
\hline Intercept & 0.953 & 0.000 & 2288.704 & $0.000^{* * *}$ \\
Initial population size & 0.000 & 0.000 & 6.500 & $0.000 * * *$ \\
Initial consumer resource stock & 0.000 & 0.000 & 0.446 & 0.656 \\
Maximum resource regrow rate & 0.000 & 0.000 & 19.891 & $0.000^{* * *}$ \\
Maximum resource capacity & 0.000 & 0.000 & 2.891 & $0.004 * *$ \\
\hline $\mathrm{F}(4,251)=111.6(p<0.001)$ & $\mathrm{R}^{2}=0.63$ & & &
\end{tabular}

d. Gini consumer resource stock

\begin{tabular}{lrrrl}
\hline & \multicolumn{3}{c}{ Standard } & \\
& Estimate & error & t value & \multicolumn{1}{c}{$\boldsymbol{p}$} \\
\hline Intercept & 0.283 & 0.000 & 431.434 & $0.000^{* * *}$ \\
Initial population size & 0.000 & 0.000 & 3.472 & $0.001^{* * *}$ \\
Initial consumer resource stock & 0.000 & 0.000 & 0.154 & 0.876 \\
Maximum resource regrow rate & 0.000 & 0.000 & -2.267 & $0.024^{*}$ \\
Maximum resource capacity & 0.000 & 0.000 & -38.517 & $0.000^{* * *}$ \\
\hline
\end{tabular}

$\mathrm{F}(4,251)=375.2(p<0.001) \quad \mathrm{R}^{2}=0.85$ 
e. Final population size

982

\begin{tabular}{|c|c|c|c|c|}
\hline & \multicolumn{3}{|c|}{ Standard } & \multirow{2}{*}{$\begin{array}{r}983 \\
\boldsymbol{p}_{0} \\
\end{array}$} \\
\hline & Estimate & error & $t$ value & \\
\hline \multirow{2}{*}{ Intercept } & - & 188.701 & -14.773 & \\
\hline & 2787.749 & & & 0.000985 \\
\hline Initial population size & 2.496 & 0.118 & 21.110 & 0.000 敞类 \\
\hline Initial consumer resource stock & 0.589 & 2.738 & 0.215 & 0.830 987 \\
\hline Maximum resource regrow rate & 191.341 & 8.038 & 23.805 & 0.000 * \\
\hline Maximum resource capacity & 61.961 & 2.738 & 22.634 & $0.000 \gg 88$ \\
\hline \multirow[t]{2}{*}{$\mathrm{F}(4,251)=381.2(p<0.001)$} & $R^{2}=0.86$ & & & 989 \\
\hline & & & & 990 \\
\hline
\end{tabular}

\section{Appendix 4. Calculation of Entropy}

992 The entropy of the consumer resource stocks was calculated as the Shannon index, or Shannon 993 entropy, of the resource stocks held by consumers. As the Shannon entropy is meant to be applied 994 to discrete data, the consumer resource stocks were discretised into a fixed number of 'bins' using 995 Sturges' formula (Sturges, 1926), and the Shannon entropy was calculated for the bins.

996 Sturges' formula for the number of bins $k$ for a population of size $n$ is

$997 \mathrm{k}=\left\lceil\log _{2} \mathrm{n}\right\rceil+1$.

998 Using a sample of 100 runs from the stability test for run length (Section A3), the normality of the 999 consumer resource stocks at the sampling timesteps $T=500, T=750$, and $T=1000$ was tested. 1000 Additionally, the entropy was calculated for 5, 10, 15, 20, 25, 50, 75, and 100 bins and compared 1001 with the entropy binned using Sturges' formula. By normalising the calculated entropy by the 1002 maximum possible entropy for that number of bins, $\log (N)$, the differences in entropy between 1003 different numbers of bins were $<0.001$. As the data were found to be approximately normally 1004 distributed at the sampling timesteps, the assumptions for Sturges' formula was met, and it was 1005 chosen to determine the final bin width. 


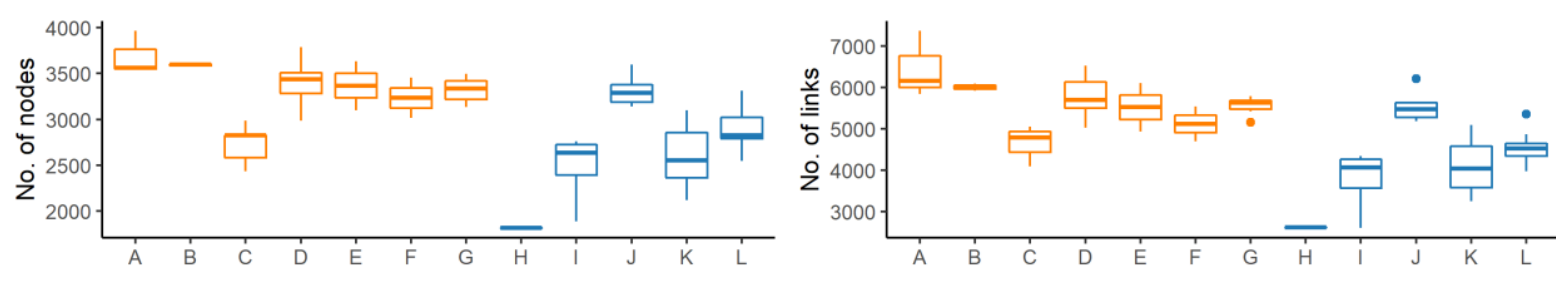

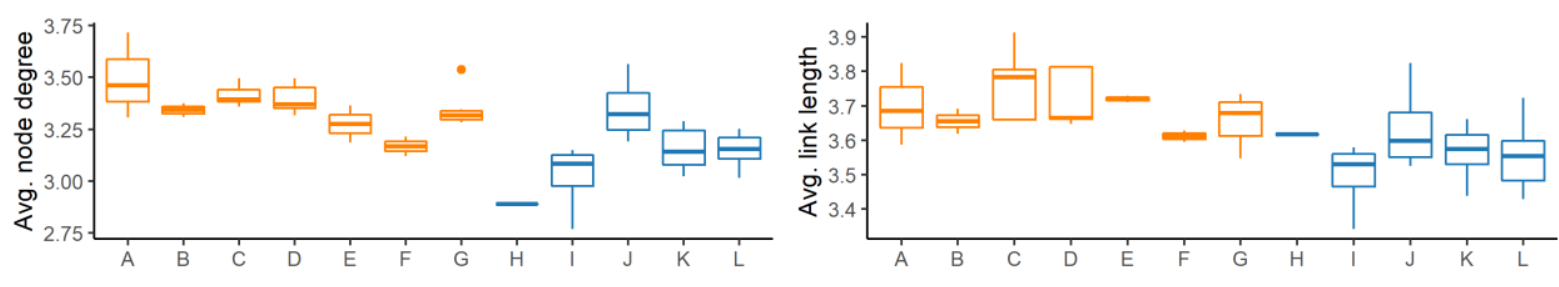

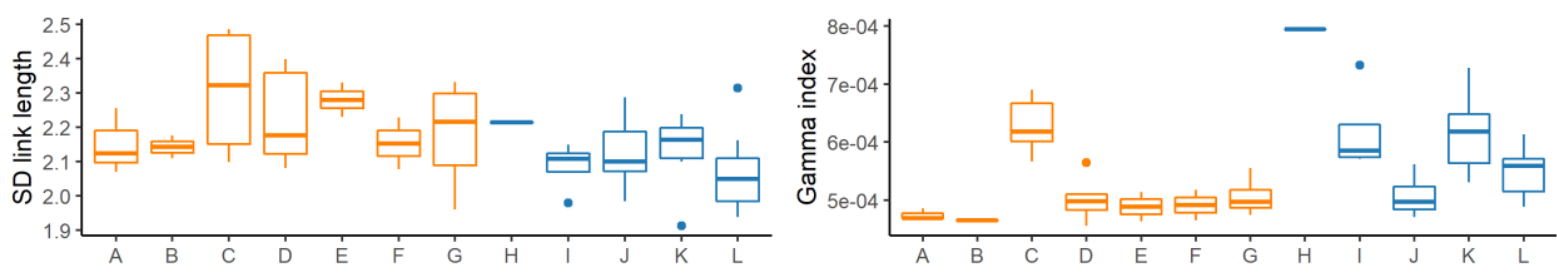

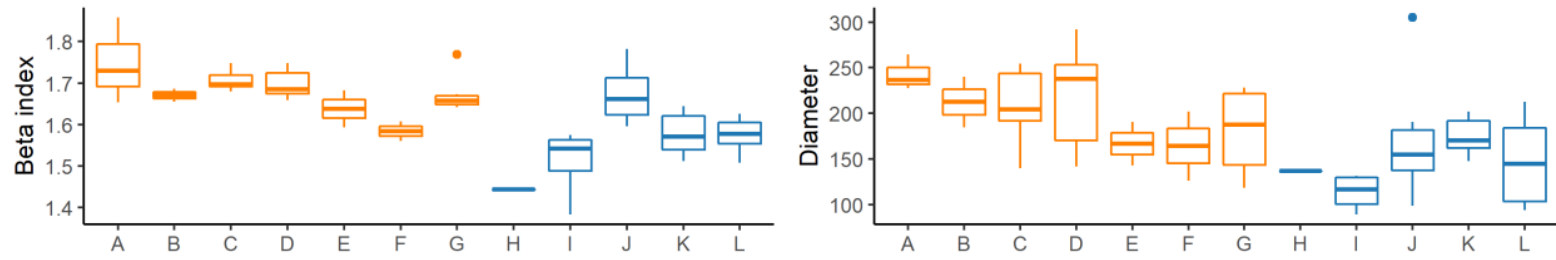

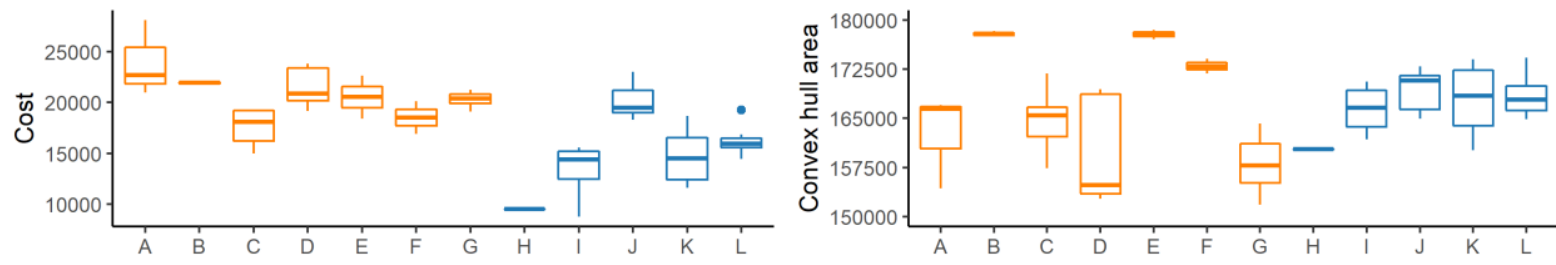

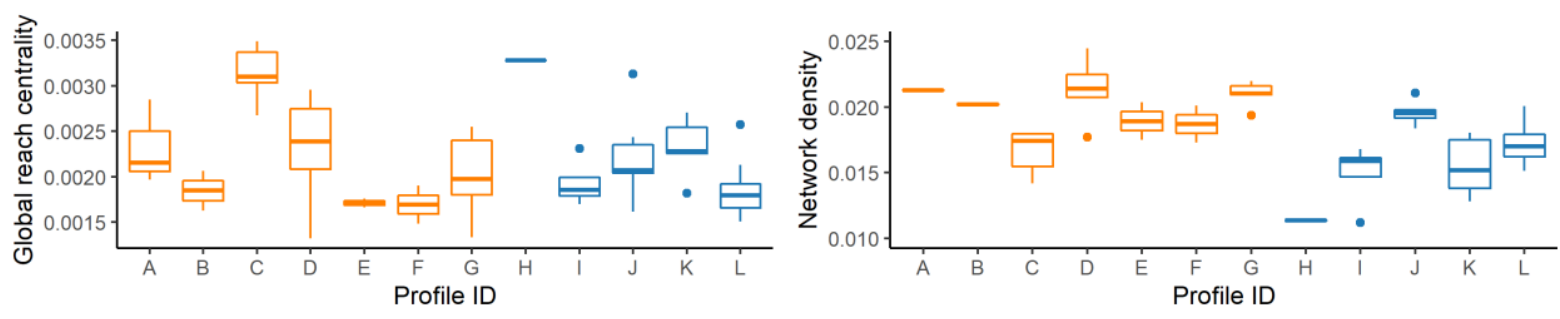

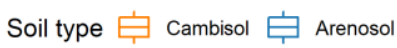
a. 

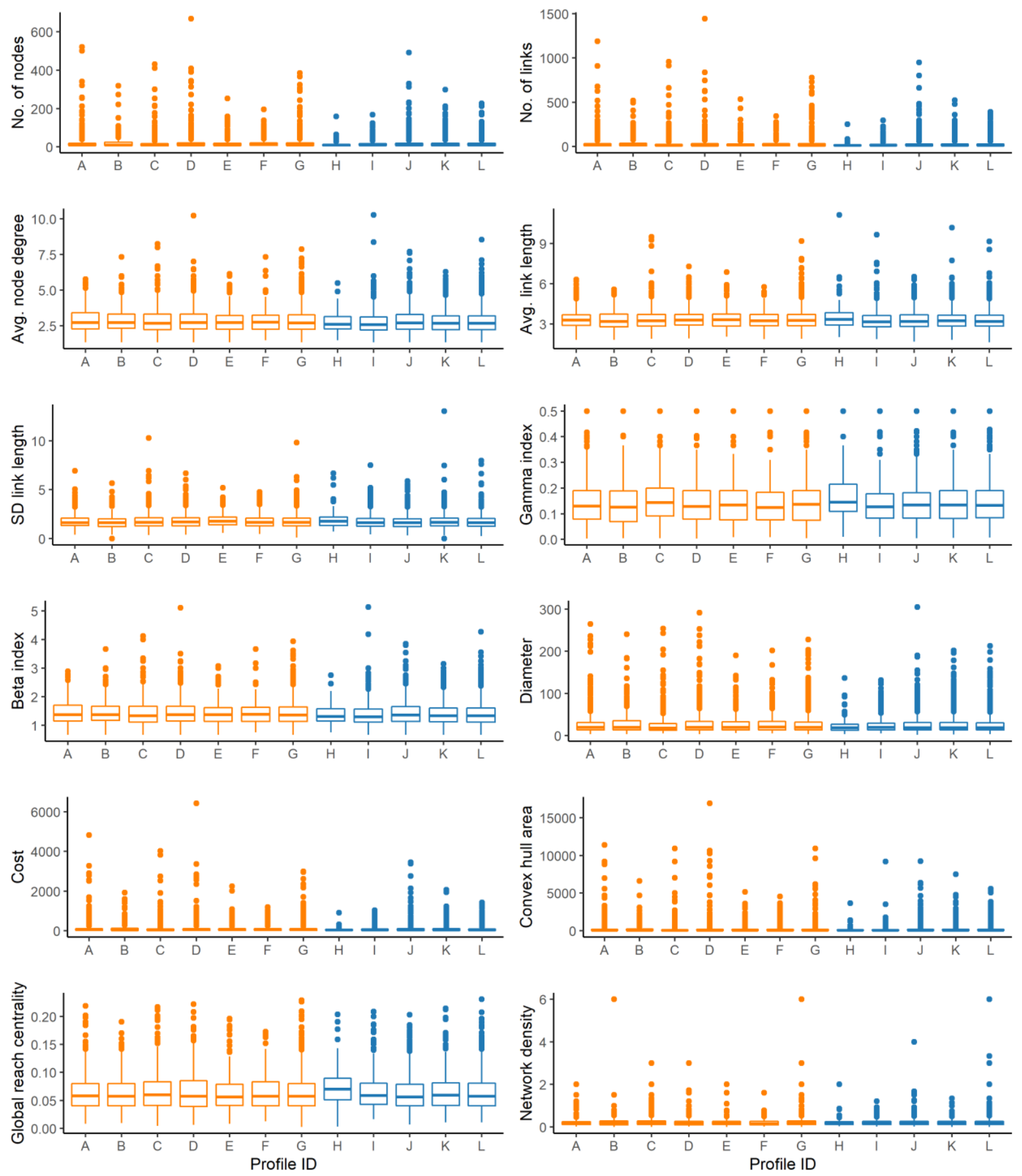

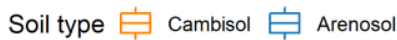

b.

Figure A7. Boxplots showing distributions of network metrics across soil profiles for (a) main are Arenosol soil profiles. Descriptions of network metrics are in Table 1. 